\title{
Tadpoles and commutative spectral triples
}

\author{
Bruno Iochum and Cyril Levy
}

\begin{abstract}
Using the Chamseddine-Connes approach of the noncommutative action on spectral triples, we show that there are no tadpoles of any order for compact spin manifolds without boundary, and also consider a case of a chiral boundary condition. Using pseudodifferential techniques, we study noncommutative integrals in commutative geometries.
\end{abstract}

PACS numbers. 11.10.Nx, 02.30.Sa, 11.15.Kc.

Mathematics Subject Classification (2010). 46H35, 46L52, 58B34.

Keywords. Noncommutative geometry, spectral triple, noncommutative residue.

\section{Introduction}

The history of noncommutative residue is now rather long [35], so we sketch it only briefly: after some approaches by Adler [2] and Manin [42] on the Korteweg-de Vries equation using a trace on the algebra of formal pseudodifferential operators in one dimension, and of Guillemin with his "soft" proof of Weyl's law on the eigenvalues of an elliptic operator [29], the noncommutative residue in any dimension was essentially initiated by Wodzicki in his thesis [50]. This residue gives the unique non-trivial trace on the algebra of pseudodifferential operators. Then a link between this residue and the Dixmier's trace was given by Connes in [10]. Due to Connes [11], [12], the setting of classical pseudodifferential operators on Riemannian manifolds without boundary was extended to a noncommutative geometry where the manifold is replaced by a not necessarily commutative algebra $\mathcal{A}$ plus a Dirac-like operator $\mathscr{D}$ via the notion of spectral triple $(\mathscr{A}, \mathscr{H}, \mathscr{D})$, where $\mathscr{H}$ is the Hilbert space acted upon by $\mathcal{A}$ and $D$. The previous Dixmier's trace is extended to the algebra of pseudodifferential operators naturally associated to the triple $(\mathcal{A}, \mathscr{H}, \mathcal{D})$. This spectral point of view appears quite natural in the general framework of noncommutative geometry which goes beyond Riemannian geometry. From a physicist point of view, this framework has many advantages: the spectral approach is motivated by quantum physics but not only since classical observables and infinitesimals are now on the same footing and even Dixmier's trace is related to renormalization. It is amazing to observe that most of the classical geometrical notions like those defined in relativity or particle physics can be extended to this really noncommutative setting. Among others, some physical 
actions still make sense as in [10], where Dixmier's trace is used to compute the Yang-Mills action in the context of noncommutative differential geometry. Another example is the Einstein-Hilbert action: on a compact spin Riemannian 4-manifold, $f D^{-2}$ coincides (up to a universal scalar) with the Einstein-Hilbert action, where $f$ is precisely the noncommutative residue, a point first noticed by Connes; then there were some brute force proof [36] and generalization [34] (see also [1]) of this fact which is particularly relevant here.

Since then the case of compact manifolds with boundary has been studied, making clearer the links between noncommutative residues, Dixmier's trace and heat kernel expansion. This was made using Boutet de Monvel's algebra [20], [46], [27], in the case of conical singularities [45], [40] or when the symbols are log-polyhomogeneous [39]. Besides, applications of noncommutative residues for such manifolds to classical gravity were found [49], and better, when the gravity is unified with fundamental interactions [8]. Needless to say that in field theory the one-loop calculation divergences, anomalies and different asymptotics of the effective action are directly obtained from the heat kernel method [48], so all of the above-mentioned mathematical results have profound applications to physics.

The Chamseddine-Connes action [7] associated to a spectral triple $(\mathcal{A}, \mathscr{H}, \mathscr{D})$ is, for a 1-form $A=\sum_{i} a_{i}\left[\mathscr{D}, b_{i}\right], a_{i}, b_{i} \in \mathcal{A}$,

$$
S\left(D_{A}, \Phi, \Lambda\right)=\sum_{0<k \in \mathrm{Sd}^{+}} \Phi_{k} \Lambda^{k} f\left|\mathscr{D}_{A}\right|^{-k}+\Phi(0) \zeta_{D_{A}}(0)+\mathcal{O}\left(\Lambda^{-1}\right),
$$

where $\mathscr{D}_{A}:=\mathscr{D}+A\left(\right.$ or $\mathscr{D}_{\tilde{A}}:=\mathscr{D}+\tilde{A}, \tilde{A}:=A+\epsilon J A J^{-1}$ in the real case), $\Phi_{k}=\frac{1}{2} \int_{0}^{\infty} \Phi(t) t^{k / 2-1} d t$ and $\mathrm{Sd}^{+}$is the strictly positive part of the dimension spectrum of the spectral triple. When $\mathscr{D}_{A}$ is not invertible, we invert in (1) the invertible operator $\mathscr{D}_{A}+P_{A}$, where $P_{A}$ is the projection on Ker $\mathscr{D}_{A}$ which is a finite dimensional space.

The coefficient $\zeta_{D_{A}}(0)$ related to the constant term in (1) can be computed from the unperturbed spectral action since it was proved in [16] (with an invertible Dirac operator and a 1 -form $A$ such that $\mathscr{D}+A$ is also invertible) that

$$
\zeta_{\mathcal{D}+A}(0)-\zeta_{\mathcal{D}}(0)=\sum_{q=1}^{n} \frac{(-1)^{q}}{q} f\left(A D^{-1}\right)^{q},
$$

using $\zeta_{X}(s)=\operatorname{Tr}\left(|X|^{-s}\right)$.

It is important to be able to compute (1) and here we look at the possible cancellation of terms in this formula. We focus essentially on commutative spectral triples, where we show that there are no tadpoles, i.e., terms like $f A D^{-1}$ are zero: in field theory, $\mathscr{D}^{-1}$ is the Feynman propagator and $A D^{-1}$ is a one-loop graph with fermionic internal line and only one external bosonic line $A$ looking like a tadpole. More generally, the tadpoles are the $A$-linear terms in (1). 


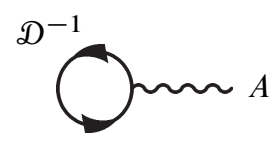

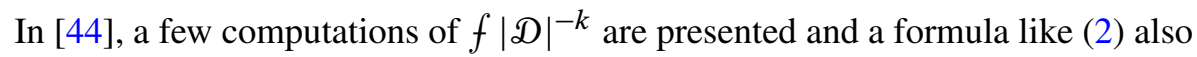
appears in [41] in the context of pseudodifferential elliptic operators.

For examples of spectral action in the real noncommutative setting, see [9], [6], [37] for the case of almost commutative instances which pops up in particle physics, [21] for the Moyal plane (and few points for non-compact manifolds [22]), [23], [19] for the noncommutative torus, and [33] for the quantum group $\mathrm{SU}_{q}(2)$. In the latter case there are tadpoles.

As a starting point, we investigate in Section 2 the existence of tadpoles for manifolds with boundaries and consider, following Chamseddine and Connes [8], the case of a chiral boundary condition on the Dirac operator. One of Chamseddine and Connes' original motivations was to show that the first two terms in spectral action come with the right ratio and sign for their coefficients as in the modified Euclidean action used in gravitation. We generalize this approach to the perturbed Dirac operator by an internal fluctuation, ending up with no tadpoles up to order 5 (see Definition 3.4).

However, this approach stems from explicit computations of first heat kernel coefficients, so we cannot conclude that other integrals of the same type as tadpoles are zero. It is then natural to restrict to manifolds without boundary via a different method.

We gather in Section 3 some basic results concerning the use of the reality operator $J$. We use these results to prove Theorem 4.3 in Section 4.2, which asserts that there are no tadpoles of any order in commutative geometries. After some useful facts using the link between $f$ and the Wodzicki residue, we study in Section 4.3 some tadpole-like noncommutative integrals, and prove Theorem 4.6, which extends Theorem 4.3. Finally, we conclude in Section 4.4 with a few remarks about nonlinear terms appearing in the spectral action (1).

Technical proofs and a few definitions about pseudodifferential operators, dimension spectrum are postponed to the Appendix.

\section{Tadpoles and compact spin manifolds with boundary}

Let $M$ be a smooth compact Riemannian $d$-dimensional manifold with smooth boundary $\partial M$ and $V$ be a given smooth vector bundle on $M$. We denote by $d x$ (resp. $d y$ ) the Riemannian volume form on $M$ (resp. on $\partial M$ ).

Recall that a differential operator $P$ is of Laplace type if it has locally the form

$$
P=-\left(g^{\mu v} \partial_{\mu} \partial_{\nu}+\mathbb{A}^{\mu} \partial_{\mu}+\mathbb{B}\right)
$$


where $\left(g^{\mu \nu}\right)_{1 \leq \mu, \nu \leq d}$ is the inverse matrix associated to the metric $g$ on $M$, and $\mathbb{A}^{\mu}$ and $\mathbb{B}$ are smooth $L(V)$-sections on $M$ (endomorphisms). A differential operator $D$ is of Dirac type if $D^{2}$ is of Laplace type, or equivalently if it has locally the following form

$$
D=-i \gamma^{\mu} \partial_{\mu}+\phi
$$

where $\left(\gamma^{\mu}\right)_{1 \leq \mu \leq d}$ gives $V$ a Clifford module structure: $\left\{\gamma^{\mu}, \gamma^{\nu}\right\}=2 g^{\mu \nu} \operatorname{Id}_{V}$, $\left(\gamma^{\mu}\right)^{*}=\gamma^{\mu}$.

A particular case of Dirac operator is given by the following formula

$$
D=-i \gamma^{\mu}\left(\partial_{\mu}+\omega_{\mu}\right)
$$

where the $\omega_{\mu}$ are in $C^{\infty}(L(V))$.

If $P$ is a Laplace type operator of the form (3), then (see [25], Lemma 1.2.1) there is a unique connection $\nabla$ on $V$ and a unique endomorphism $E$ such that $P=L(\nabla, E)$, where by definition

$$
L(\nabla, E):=-\left(\operatorname{Tr}_{g} \nabla^{2}+E\right), \quad \nabla^{2}(X, Y):=\left[\nabla_{X}, \nabla_{Y}\right]-\nabla_{\nabla_{X}^{g} Y},
$$

$X, Y$ are vector fields on $M$ and $\nabla^{g}$ is the Levi-Civita connection on $M$. Locally

$$
\operatorname{Tr}_{g} \nabla^{2}:=g^{\mu \nu}\left(\nabla_{\mu} \nabla_{\nu}-\Gamma_{\mu \nu}^{\rho} \nabla_{\rho}\right),
$$

where $\Gamma_{\mu \nu}^{\rho}$ are the Christoffel coefficients of $\nabla^{g}$. Moreover (with local frames of $T^{*} M$ and $\left.V\right), \nabla=d x^{\mu} \otimes\left(\partial_{\mu}+\omega_{\mu}\right)$ and $E$ are related to $g^{\mu \nu}, \mathbb{A}^{\mu}$ and $\mathbb{B}$ through

$$
\begin{aligned}
\omega_{\nu} & =\frac{1}{2} g_{\nu \mu}\left(\mathbb{A}^{\mu}+g^{\sigma \varepsilon} \Gamma_{\sigma \varepsilon}^{\mu} \mathrm{Id}\right), \\
E & =\mathbb{B}-g^{\nu \mu}\left(\partial_{\nu} \omega_{\mu}+\omega_{\nu} \omega_{\mu}-\omega_{\sigma} \Gamma_{\nu \mu}^{\sigma}\right) .
\end{aligned}
$$

Suppose that $P=L(\nabla, E)$ is a Laplace type operator on $M$, and assume that $\chi$ is an endomorphism of $V_{\partial M}$ so that $\chi^{2}=\operatorname{Id}_{V}$. We extend $\chi$ on a collar neighborhood $\mathcal{C}$ of $\partial M$ in $M$ with the condition $\nabla_{d}(\chi)=0$, where the $d$-th coordinate here is the radial coordinate (the geodesic distance of a point in $M$ to the boundary $\partial M$.)

Let $V_{ \pm}:=\Pi_{ \pm} V$ the sub-bundles of $V$ on $\ell$ where $\Pi_{ \pm}:=\frac{1}{2}\left(\operatorname{Id}_{V} \pm \chi\right)$ are the projections on the \pm 1 eigenvalues of $\chi$. We also fix an auxiliary endomorphism $S$ on $V_{+\partial M}$ extended to $C$.

This allows to define the mixed boundary operator $\mathscr{B}=\mathscr{B}(\chi, S)$ as

$$
\mathscr{B} s:=\Pi_{+}\left(\nabla_{d}+S\right) \Pi_{+} s_{\mid \partial M} \oplus \Pi_{-} s \mid \partial M, \quad s \in C^{\infty}(V) .
$$

These boundary conditions generalizes Dirichlet $\left(\Pi_{-}=\operatorname{Id}_{V}\right.$, so $\left.\chi=-\operatorname{Id}_{V}\right)$ and Neumann-Robin $\left(\Pi_{+}=\mathrm{Id}_{V}\right.$, so $\left.\chi=\operatorname{Id}_{V}\right)$ conditions.

We define $P_{\mathscr{B}}$ as the realization of $P$ on $\mathcal{B}$, that is to say the closure of $P$ defined on the space of smooth sections of $V$ satisfying the boundary condition $\mathscr{B} s=0$. 
We are interested in the behavior of heat kernel coefficients $a_{d-n}$ defined through its expansion as $\Lambda \rightarrow \infty$ (see [25], Theorem 1.4.5)

$$
\operatorname{Tr}\left(e^{-\Lambda^{-2} D_{\mathcal{B}}^{2}}\right) \sim \sum_{n \geq 0} \Lambda^{d-n} a_{d-n}(D, \mathscr{B}),
$$

where $D$ is a self-adjoint Dirac type operator. Moreover, we will use a perturbation $D \rightarrow D+A$, where $A$ is a 1 -form (a linear combination of terms of the type $f[D, g]$, where $f$ and $g$ are smooth functions on $M$ ). More precisely, we investigate the linear dependence of these coefficients with respect to $A$. It is clear that, since $A$ is differential operator of order 0 , a perturbation $D \mapsto D+A$ transforms a Dirac type operator into another Dirac type operator.

This perturbation has consequences on the $E$ - and $\nabla$-terms:

Lemma 2.1 ([48]). Let D be a Dirac type operator locally of the form (4) such that $\nabla_{\mu}:=\partial_{\mu}+\omega_{\mu}$ is connection compatible with the Clifford action $\gamma$. Let A be a 1 -form associated to $D$ so that $A$ is locally of the form $-i \gamma^{\mu} a_{\mu}$ with $a_{\mu} \in C^{\infty}(U)$, $\left(U, x_{\mu}\right)$ being a local coordinate frame on $M$.

Then $(D+A)^{2}=L\left(\nabla^{A}, E^{A}\right)$ and $D^{2}=L(\nabla, E)$, where

$$
\begin{gathered}
\omega_{\mu}^{A}=\omega_{\mu}+a_{\mu}, \text { thus } \nabla_{\mu}^{A}=\nabla_{\mu}+a_{\mu} \operatorname{Id}_{V}, \\
E^{A}=E+\frac{1}{4}\left[\gamma^{\mu}, \gamma^{\nu}\right] F_{\mu \nu}, \quad E=\frac{1}{2} \gamma^{\mu} \gamma^{\nu}\left[\nabla_{\mu}, \nabla_{\nu}\right], \quad F_{\mu \nu}:=\partial_{\mu}\left(a_{\nu}\right)-\partial_{\nu}\left(a_{\mu}\right) .
\end{gathered}
$$

Moreover, the curvature of the connection $\nabla^{A}$ is $\Omega_{\mu \nu}^{A}=\Omega_{\mu \nu}+F_{\mu \nu}$, where $\Omega_{\mu \nu}=$ $\left[\nabla_{\mu}, \nabla_{\nu}\right]$.

In particular $\operatorname{Tr} E^{A}=\operatorname{Tr} E$.

Observe that even if quadratic terms in $A^{2}$ appear in the local presentation of the perturbation $D^{2} \rightarrow(D+A)^{2}$ (in the $b$-term), these terms do not appear in the invariant formulation $(\nabla, E)$ since they are hidden in $\nabla_{\mu}^{A} \nabla_{\nu}^{A}$.

In the following, $D$ and $A$ are fixed and satisfy the hypothesis of Lemma 2.1. Indices $i, j, k$, and $l$ range from 1 through the dimension $d$ of the manifold and index a local orthonormal frame $\left\{e_{1}, \ldots, e_{d}\right\}$ for the tangent bundle. Roman indices $a, b, c$, range from 1 through $d-1$ and index a local orthonormal frame for the tangent bundle of the boundary $\partial M$. The vector field $e_{d}$ is chosen to be the inward-pointing unit normal vector field. Greek indices are associated to coordinate frames.

Let $R_{i j k l}, \rho_{i j}:=R_{i k k j}$ and $\tau:=\rho_{i i}$ be the components of the Riemann tensor, Ricci tensor and scalar curvature of the Levi-Civita connection, respectively. Let $L_{a b}:=\left(\nabla_{e_{a}} e_{b}, e_{d}\right)$ be the second fundamental form of the hypersurface $\partial M$ in $M$. Let ";" denote multiple covariant differentiations with respect to $\nabla^{A}$, and let ":" denote multiple covariant differentiations with respect to $\nabla$ and the Levi-Civita connection of $M$.

We will look at a chiral boundary condition. This is a mixed boundary condition natural to consider in order to preserve the existence of chirality on $M$ and its boundary 
$\partial M$ which are compatible with the (selfadjoint) Clifford action: we assume that the operator $\chi$ is selfadjoint and satisfies the relations

$$
\left\{\chi, \gamma^{d}\right\}=0, \quad\left[\chi, \gamma^{a}\right]=0 \quad \text { for all } a \in\{1, \ldots, d-1\} .
$$

This condition was shown in [8] a natural assumption to enforce the hermiticity of the realization of the Dirac operator. It is known [25], Lemma 1.5.3, that ellipticity is preserved.

Since $\gamma^{d}$ is invertible, $\operatorname{dim} V_{+}=\operatorname{dim} V_{-}$and $\operatorname{Tr} \chi=0$.

For an even-dimensional oriented manifold, there is a natural candidate $\chi$ satisfying (6), namely

$$
\chi:=\chi_{\partial M}=(-i)^{d / 2-1} \gamma\left(e_{1}\right) \ldots \gamma\left(e_{d-1}\right)
$$

(this notation is compatible with (11)). Recall that

$$
\operatorname{Tr}\left(\gamma^{i_{1}} \ldots \gamma^{i_{2 k+1}}\right)=0 \text { for all } k \in \mathbb{N}, \quad \operatorname{Tr}\left(\gamma^{i} \gamma^{j}\right)=\operatorname{dim} V \delta^{i j} .
$$

The natural realization of this boundary condition for the Dirac type operator $D+A$ is the operator $(D+A)_{\chi}$ which acts as $D+A$ on the domain $\left\{s \in C^{\infty}(V)\right.$ : $\left.\Pi_{-} s \mid \partial M=0\right\}$. It turns out (see [4], Lemma 7) that the natural boundary operator $B_{\chi}^{A}$ defined by

$$
\mathcal{B}_{\chi}^{A} s:=\Pi_{-}(D+A) s_{\mid \partial M} \oplus \Pi_{-} s_{\mid \partial M}
$$

is a boundary operator of the form (5) provided that $S=\frac{1}{2} \Pi_{+}\left(-i\left[\gamma^{d}, A\right]-L_{a a} \chi\right) \Pi_{+}$.

Lemma 2.2. Actually, $S$ and $\chi_{; a}$ are independent of the perturbation $A$ :

(i) $S=-\frac{1}{2} L_{a a} \Pi_{+}$.

(ii) $\chi ; a=\chi: a$.

Proof. (i) Since $A$ is locally of the form $-i \gamma^{j} a_{j}$ with $a_{j} \in C^{\infty}(U)$, we obtain from (6)

$\chi\left[\gamma^{d}, A\right]=-i a_{j} \chi\left[\gamma^{d}, \gamma^{j}\right]=-i \sum_{j<d} a_{j} \chi\left[\gamma^{d}, \gamma^{j}\right]=i \sum_{j<d} a_{j}\left[\gamma^{d}, \gamma^{j}\right] \chi=-\left[\gamma^{d}, A\right] \chi$ and the result as a consequence of $\Pi_{+}\left[\gamma^{d}, A\right]=\left[\gamma^{d}, A\right] \Pi_{-}$and $\Pi_{+} \Pi_{-}=0$.

(ii) We have $\nabla_{i}^{A}=\nabla_{i}+a_{i} \operatorname{Id}_{V}$ where $A=:-i \gamma^{j} a_{j}$, and since $\left(\nabla_{i}^{A} \chi\right) s=$ $\nabla_{i}^{A}(\chi s)-\chi\left(\nabla_{i}^{A} s\right)$ for any $s \in C^{\infty}(V)$, it follows Lemma 2.1 from that $\nabla_{i}^{A}(\chi)=$ $\left[\nabla_{i}+a_{i} \operatorname{Id}_{V}, \chi\right]=\left[\nabla_{i}, \chi\right]=\nabla_{i}(\chi)$.

While $S$ is not sensitive to the perturbation $A$, the boundary operator $\mathscr{B}_{\chi}^{A}$ depends a priori on $A$. We shall denote $\mathscr{B}_{\chi}$ the boundary operator $\mathcal{B}_{\chi}^{A}$ when $A=0$.

The coefficients $a_{d-k}$ for $0 \leq k \leq 4$ have been computed in [3] for general mixed boundary conditions in the case of Laplace type operators and in [4], Lemma 8, for Dirac type operators with chiral boundary conditions. We recall here these coefficients in our setting: 


\section{Proposition 2.3.}

$$
\begin{aligned}
& a_{d}\left(D+A, \mathcal{B}_{\chi}^{A}\right)=(4 \pi)^{-d / 2} \int_{M} \operatorname{Tr}_{V} 1 d x, \\
& a_{d-1}\left(D+A, B_{\chi}^{A}\right)=0 \text {, } \\
& a_{d-2}\left(D+A, \mathscr{B}_{\chi}^{A}\right)=\frac{(4 \pi)^{-d / 2}}{6}\left\{\int_{M} \operatorname{Tr}_{V}\left(6 E^{A}+\tau\right) d x\right. \\
& \left.+\int_{\partial M} \operatorname{Tr}_{V}\left(2 L_{a a}+12 S\right) d y\right\} \\
& a_{d-3}\left(D+A, \mathcal{B}_{\chi}^{A}\right)=\frac{(4 \pi)^{-(d-1) / 2}}{384} \int_{\partial M} \operatorname{Tr}_{V}\left\{96 \chi E^{A}+3 L_{a a}^{2}+6 L_{a b}^{2}\right. \\
& \left.+96 S L_{a a}+192 S^{2}-12 \chi_{; a}^{2}\right\} d y, \\
& a_{d-4}\left(D+A, \mathcal{B}_{\chi}^{A}\right)=\frac{(4 \pi)^{-d / 2}}{360}\left\{\int _ { M } \operatorname { T r } _ { V } \left\{60 \tau E^{A}+180\left(E^{A}\right)^{2}+30\left(\Omega_{i j}^{A}\right)^{2}\right.\right. \\
& \left.+5 \tau^{2}-2 \rho^{2}+2 R^{2}\right\} d x \\
& +\int_{\partial M} \operatorname{Tr}_{V}\left\{180 \chi E_{; d}^{A}+120 E^{A} L_{a a}+720 S E^{A}\right. \\
& \left.\left.+60 \chi \chi_{; a} \Omega_{a d}^{A}+T\right\} d y\right\} .
\end{aligned}
$$

where

$$
\begin{aligned}
T:= & 20 \tau L_{a a}+4 R_{a d a d} L_{b b}-12 R_{a d b d} L_{a b}+4 R_{a b c b} L_{a c} \\
& +\frac{1}{21}\left(160 L_{a a}^{3}-48 L_{a b}^{2} L_{c c}+272 L_{a b} L_{b c} L_{a c}\right. \\
& +120 \tau S+144 S L_{a a}^{2}+48 S L_{a b}^{2}+480\left(S^{2} L_{a a}+S^{3}\right) \\
& \left.-42 \chi_{; a}^{2} L_{b b}+6 \chi_{; a} \chi_{; b} L_{a b}-120 \chi_{; a}^{2} S\right)
\end{aligned}
$$

is independent of $A$.

The following proposition shows that there are no tadpoles (linear terms in $A$ ) in the heat kernel coefficients $a_{d-k}\left(D+A, \mathscr{B}_{\chi}^{A}\right)$ for $k \leq 5$ in the case of manifolds endowed with a chiral boundary condition.

Theorem 2.4. Let $M$ be an even d-dimensional compact oriented spin Riemannian manifold with smooth boundary $\partial M$ and spin bundle $V$. Let $D:=-i \gamma^{j} \nabla_{j}$ be the classical Dirac operator and $\chi=\chi_{\partial M}=(-i)^{d / 2-1} \gamma\left(e_{1}\right) \ldots \gamma\left(e_{d-1}\right)$, where $\left(e_{i}\right)_{1 \leq i \leq d}$ is a local orthonormal frame of TM.

The perturbation $D \rightarrow D+A$, where $A=-i \gamma^{j} a_{j}$ is a 1-form for $D$, induces, under the chiral boundary condition, the following perturbations on the heat kernel coefficients, where we set $c_{d-k}(A):=a_{d-k}\left(D+A, \mathscr{B}_{\chi}^{A}\right)-a_{d-k}\left(D, B_{\chi}\right)$ : 
(i) $c_{d}(A)=c_{d-1}(A)=c_{d-2}(A)=c_{d-3}(A)=0$,

(ii) $c_{d-4}(A)=-\frac{1}{6(2 \pi)^{d / 2}} \int_{M} F_{\mu \nu} F^{\mu v} d x$.

In other words, the coefficients $a_{d-k}$ for $0 \leq k \leq 3$ are unperturbed; $a_{d-4}$ is only perturbed by quadratic terms in $A$, and there are no A-linear terms in $a_{d-k}(D+$ $\left.A, B_{\chi}^{A}\right)$ for $k \leq 5$.

Remark 2.5. We conjecture that there are no $A$-linear terms in the heat kernel coefficients $a_{d-k}\left(D+A, B_{\chi}^{A}\right)$ for any $k \in \mathbb{N}$. Since we want to work with a selfadjoint realization $(D+A)_{\chi}$, the chiral boundary condition was chosen. This condition corresponds to a realization acting as $D+A$ on the domain $\{\psi: \chi(\psi)=-\psi\}$. As we saw, this condition yields on the square $\left((D+A)_{\chi}\right)^{2}$ a mixed boundary condition: $\left((D+A)_{\chi}\right)^{2}=\left((D+A)^{2}\right)_{\mathcal{B}_{\chi}^{A}}$ where $\mathcal{B}_{\chi}^{A}$ is the mixed boundary operator given in (8), with a fixed endomorphism $S$. In particular, $S$ is fixed by the chiral condition on $D+A$. In other words, there is no other possible choice for $S$.

Note that the Dirichlet condition $\left(\chi=-\mathrm{id}_{V}\right)$ and Neumann-Robin $\left(\chi=\operatorname{id}_{V}\right)$ would not yield a selfadjoint realization since (6) would not be satisfied in this case.

Remark 2.6. If $A$ is selfadjoint, all coefficients $a_{d-k}\left(D+A, \mathscr{B}_{\chi}^{A}\right)$ and $a_{d-k}\left(D, \mathscr{B}_{\chi}\right)$ are real, while linear contributions in $A$ are purely imaginary, modulo traces of $\gamma$ and $\chi$-matrices and their covariant derivatives. Since the invariant terms appearing as integrands of $\int_{M}$ and $\int_{\partial M}$ in the coefficients at higher order are polynomial in $S, \chi$, $R, E^{A}$ and $\Omega^{A}$, and their covariant derivatives, one expects no linear terms in $A$ at any order.

We study more examples in [32] with a generalization of Theorem 4.3.

Proof. (i) The fact that $c_{d}(A)=c_{d-1}(A)=0$ follows from Proposition 2.3.

Since by Lemma 2.2, $c_{d-2}(A)=(4 \pi)^{-d / 2} \int_{M} \operatorname{Tr}_{V}\left(E^{A}-E_{A}\right) d x$, it follows that $c_{d-2}(A)=0$ because $\operatorname{Tr}_{V} E^{A}=\operatorname{Tr}_{V} E$ by Lemma 2.1.

From Proposition 2.3 and Lemma 2.2, we get

$$
c_{d-3}(A)=\frac{1}{4}(4 \pi)^{-(d-1) / 2} \int_{\partial M} \operatorname{Tr}_{V}\left\{\chi\left(E^{A}-E\right)\right\} .
$$

Since $\chi\left(E^{A}-E\right)=(-i)^{d / 2} \gamma^{1} \ldots \gamma^{d-1}\left[\gamma^{j}, \gamma^{k}\right] F_{j k}$, (7) yields $\operatorname{Tr}_{V} \chi\left(E^{A}-E\right)=0$ because $d$ is even.

(ii) Since $\operatorname{Tr}_{V}\left(E^{A}-E\right)=0$ and $\operatorname{Tr}_{V} \chi\left(E^{A}-E\right)=0$, Lemma 2.2 implies that $\operatorname{Tr}_{V} S\left(E^{A}-E\right)=0$. Thus, using Proposition 2.3 and Lemma 2.2,

$$
\begin{aligned}
c_{d-4}(A)=\frac{(4 \pi)^{-d / 2}}{360}\{ & \int_{M} \operatorname{Tr}_{V}\left\{180\left(\left(E^{A}\right)^{2}-E^{2}\right)+30\left(\left(\Omega_{i j}^{A}\right)^{2}-\left(\Omega_{i j}\right)^{2}\right)\right\} d x \\
& \left.+\int_{\partial M} \operatorname{Tr}_{V}\left\{180 \chi\left(E_{; d}^{A}-E_{: d}\right)+60 \chi \chi_{; a}\left(\Omega_{a d}^{A}-\Omega_{a d}\right)\right\} d y\right\} .
\end{aligned}
$$


We obtain locally $\operatorname{Tr}_{V}\left(\left(E^{A}\right)^{2}-E^{2}\right)=\frac{1}{16} \operatorname{Tr}\left(\left[\gamma^{\mu}, \gamma^{\nu}\right]\left[\gamma^{\rho}, \gamma^{\sigma}\right]\right) F_{\mu \nu} F_{\rho \sigma}$ using Lichnérowicz's formula $E=-\frac{1}{4} \tau$. Since $\operatorname{Tr}_{V}\left(\left[\gamma^{\mu}, \gamma^{\nu}\right]\left[\gamma^{\rho}, \gamma^{\sigma}\right]\right)=4 \cdot 2^{d / 2}\left(g^{\mu \sigma} g^{\nu \rho}-\right.$ $\left.g^{\mu \rho} g^{\nu \sigma}\right)$, we have

$$
\operatorname{Tr}_{V}\left(\left(E^{A}\right)^{2}-E^{2}\right)=-2^{d / 2-1} F_{\mu \nu} F^{\mu \nu} .
$$

$\nabla$ being the spin connection associated to the spin structure of $M$, we have $\Omega_{i j}=$ $\frac{1}{4} \gamma^{k} \gamma^{l} R_{i j k l}$. So $R_{i j k l}=-R_{i j l k}$ implies that $\operatorname{Tr}_{V} \Omega_{i j}=0$. Hence, by Lemma 2.1,

$$
\operatorname{Tr}_{V}\left(\left(\Omega_{i j}^{A}\right)^{2}-\Omega_{i j}^{2}\right)=2^{d / 2} F_{i j}^{2}=2^{d / 2} F_{\mu \nu} F^{\mu \nu} .
$$

Also, $E_{; d}^{A}=\left[\nabla_{d}+a_{d}, E^{A}\right]=\left[\nabla_{d}, E+\frac{1}{4}\left[\gamma^{i}, \gamma^{j}\right] F_{i j}\right]=E_{: d}+\frac{1}{4}\left[\nabla_{d},\left[\gamma^{i}, \gamma^{j}\right]\right] F_{i j}$.

Using $\left[\nabla_{i}, \gamma^{i}\right]=\gamma\left(\nabla_{i} e_{j}\right)$ and (7), we obtain

$\operatorname{Tr}_{V}\left(\chi\left(E_{; d}^{A}-E_{: d}\right)\right)=(-i)^{d / 2} \frac{1}{2} F_{i j} \operatorname{Tr}_{V}\left\{\gamma^{1} \ldots \gamma^{d-1}\left(\gamma\left(\nabla_{d} e_{i}\right) \gamma^{j}+\gamma^{i} \gamma\left(\nabla_{d} e_{j}\right)\right)\right\}=0$.

It remains to check that $\operatorname{Tr}_{V}\left(\chi \chi: a\left(\Omega_{a d}^{A}-\Omega_{a d}\right)\right)=0$. Let $\chi_{M}=-i \chi \gamma^{d}$ be the grading operator (see (11).) Since $\chi_{M}$ commutes with the spin connection operator $\nabla$ (see [26], p. 396),

$$
0=\left[\nabla_{a}, \chi_{M}\right]=\left[\nabla_{a}, \chi \gamma^{d}\right]=\chi: a \gamma^{d}+\chi\left[\nabla_{a}, \gamma^{d}\right]=\chi: a \gamma^{d}+\chi \gamma\left(\nabla_{a} e_{d}\right)
$$

and thus $\chi \chi_{: a}=-\gamma\left(\nabla_{a} e_{d}\right) \gamma^{d}=-\Gamma_{a d}^{j} \gamma^{j} \gamma^{d}$, where $\Gamma_{a d}^{j}=-\Gamma_{a j}^{d}$ since $\left(e_{j}\right)$ is an

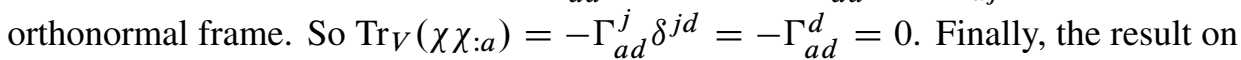
$c_{d-4}$ follows from Lemma 2.1 as $\operatorname{Tr}_{V}\left(\chi \chi: a\left(\Omega_{a d}^{A}-\Omega_{a d}\right)\right)=\operatorname{Tr}_{V}(\chi \chi: a) F_{a d}$.

The coefficient $a_{d-5}\left(D+A, \mathscr{B}_{\chi}^{A}\right)$ is computed in [5]. One can check directly as above that the linear terms in $A$ are not present. The computation uses the fact that the traces of the terms $\chi E_{; d d}^{A}, E_{; d}^{A} S, \chi\left(E^{A}\right)^{2}, E^{A} S^{2}, \chi_{; a} \chi_{; b} \Omega_{a b}^{A}, \chi_{; a}^{2} E^{A}$ do not have linear terms in $A$.

In the following, we prove the above conjecture for compact spin manifolds without boundary using Connes-Chamseddine's pseudodifferential calculus. We also see, using the Wodzicki residue, how to compute some noncommutative (tadpole-like) integrals in this setting.

\section{Tadpoles in spectral triples}

Let $(\mathcal{A}, \mathcal{D}, \mathscr{H})$ be a spectral triple of dimension $d$.

Let $J$ be the reality operator (if it exists) satisfying

$$
J \mathscr{D}=\epsilon \mathscr{D} J, \quad \epsilon= \pm 1,
$$

according to the dimension: $\epsilon=+1$ when the dimension $d$ is $0,2,3,4,6,7 \bmod 8$ and $\epsilon=-1$ when $d=1,5 \bmod 8$. 
When the triple is even, we also use the chirality operator $\chi$ which is a grading on $\mathscr{H}$ and which commutes with $\mathcal{A}$, anti-commutes with $\mathscr{D}$, and also satisfies $J \chi=\epsilon^{\prime} \chi J$, where $\epsilon^{\prime}=1$ for $d=0,4 \bmod 8$ and $\epsilon^{\prime}=-1$ for $d=2,6 \bmod 8$.

Let us recall a few definitions; see [12], [13], [18], [19], [31]:

Definition 3.1. A 1 -form $A$ is a finite sum of operators like $a_{1}\left[\mathcal{D}, a_{2}\right]$ where $a_{i} \in \mathcal{A}$.

The set of 1-forms is denoted by $\Omega_{\mathscr{D}}^{1}(\mathcal{A})$.

For definitions about the algebra $\Psi(\mathcal{A})$ of pseudodifferential operators, zeta functions and dimension spectrum, we refer the reader to the Appendix.

A. Connes introduced the notation

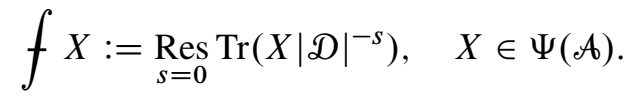

$f$ is a trace on $\Psi(\mathcal{A})$ (not necessarily positive, see Lemma 4.14.)

We assume $\mathscr{D}$ to be invertible since otherwise one can replace $\mathscr{D}$ with the invertible operator $\mathcal{D}+P, P$ being the projection on Ker $\mathcal{D}$. This change does not modify the computation of the integrals $f$ that follow.

Lemma 3.2. Let $(\mathcal{A}, \mathcal{D}, \mathscr{H})$ be a spectral triple and $X \in \Psi(\mathcal{A})$. Then

$$
f X^{*}=\overline{f x} .
$$

If the spectral triple is real, then, for $X \in \Psi(\mathcal{A}), J X J^{-1} \in \Psi(\mathcal{A})$ and

$$
f J X J^{-1}=f X^{*}=\overline{f X} .
$$

Proof. The first result follows from (for $s$ large enough, so the operators are traceable)

$$
\left.\operatorname{Tr}\left(X^{*}|\mathscr{D}|^{-s}\right)=\operatorname{Tr}\left(\left(|\mathcal{D}|^{-\bar{s}}\right) X\right)^{*}\right)=\overline{\operatorname{Tr}\left(|\mathcal{D}|^{-\bar{s}} X\right)}=\overline{\operatorname{Tr}\left(X|\mathcal{D}|^{-\bar{s}}\right)} .
$$

The second result is due to the anti-linearity of $J, \operatorname{Tr}\left(J Y J^{-1}\right)=\overline{\operatorname{Tr}(Y)}$, and $J|\mathscr{D}|=|\mathscr{D}| J$, so

$$
\operatorname{Tr}\left(X|\mathscr{D}|^{-s}\right)=\overline{\operatorname{Tr}\left(J X|\mathscr{D}|^{-s} J^{-1}\right)}=\overline{\operatorname{Tr}\left(J X J^{-1}|\mathscr{D}|^{-\bar{s}}\right)} .
$$

Corollary 3.3. For any 1 -form $A=A^{*}$, and for $k, l \in \mathbb{N}$ we have

$$
\begin{gathered}
f A^{l} \mathscr{D}^{-k} \in \mathbb{R}, \quad f\left(A D^{-1}\right)^{k} \in \mathbb{R}, \quad f A^{l}|\mathcal{D}|^{-k} \in \mathbb{R}, \\
f \chi A^{l}|\mathcal{D}|^{-k} \in \mathbb{R}, \quad f A^{l} \mathscr{D}|\mathcal{D}|^{-k} \in \mathbb{R} .
\end{gathered}
$$


In [17], the following definition is introduced:

Definition 3.4. In $(\mathcal{A}, \mathcal{H}, \mathcal{D})$, the tadpole $\operatorname{Tad}_{\mathscr{D}+A}(k)$ of order $k$, for $k \in\{d-l$ : $l \in \mathbb{N}\}$ is the term linear in $A=A^{*} \in \Omega_{\mathscr{D}}^{1}$ in the $\Lambda^{k}$-term of (1) (considered as an infinite series) where $\mathscr{D}_{A}=\mathscr{D}+A$.

If moreover, the triple $(\mathscr{A}, \mathscr{H}, \mathscr{D}, J)$ is real, the tadpole $\operatorname{Tad}_{\mathscr{D}+\tilde{A}}(k)$ is the term linear in $A$, in the $\Lambda^{k}$-term of (1) where $\mathscr{D}_{A}=\mathscr{D}+\tilde{A}$.

Proposition 3.5. Let $(\mathcal{A}, \mathscr{H}, \mathscr{D})$ be a spectral triple of dimension $d$ with simple dimension spectrum. Then

$$
\begin{aligned}
\operatorname{Tad}_{\mathscr{D}+A}(d-k) & =-(d-k) f A \mathscr{D}|\mathscr{D}|^{-(d-k)-2} \text { for all } k \neq d, \\
\operatorname{Tad}_{\mathscr{D}+A}(0) & =-f A D^{-1} .
\end{aligned}
$$

Moreover, if the triple is real, then $\operatorname{Tad}_{\mathscr{D}+\tilde{A}}=2 \operatorname{Tad}_{\mathscr{D}+A}$.

Proof. By [19], Lemma 4.6 and Proposition 4.8, we have the following formula, for any $k \in \mathbb{N}$,

$$
\begin{aligned}
& f\left|\mathscr{D}_{A}\right|^{-(d-k)}
\end{aligned}
$$

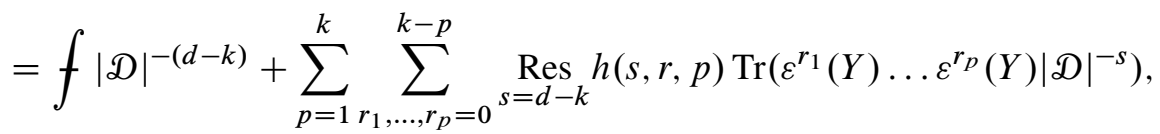

where

$$
\begin{aligned}
& h(s, r, p):=(-s / 2)^{p} \int_{0 \leq t_{1} \leq \cdots \leq t_{p} \leq 1} g\left(-s t_{1}, r_{1}\right) \ldots g\left(-s t_{p}, r_{p}\right) d t, \\
& \varepsilon^{r}(T):=\nabla(T) D^{-2 r}, \nabla(T):=\left[\mathscr{D}^{2}, T\right], \\
& g(z, r):=\left(\begin{array}{c}
z / 2 \\
r
\end{array}\right) \text { with } g(z, 0):=1, \\
& Y \sim \sum_{q=1}^{N} \sum_{k_{1}, \ldots, k_{q}=0}^{N-q} \Gamma_{q}^{k}(X) D^{-2\left(|k|_{1}+q\right)} \bmod \mathrm{OP}^{-N-1} \text { for any } \mathrm{N} \in \mathbb{N}^{*}, \\
& X:=\tilde{A} \mathscr{D}+\mathscr{D} \tilde{A}+\tilde{A}^{2}, \tilde{A}:=A+\epsilon J A J^{-1}, \\
& \Gamma_{q}^{k}(X):=\frac{(-1)^{|k|_{1}+q+1}}{|k|_{1}+q} \nabla^{k_{q}}\left(X \nabla^{k_{q-1}}\left(\ldots X \nabla^{k_{1}}(X) \ldots\right)\right) \text { for all } q \in \mathbb{N}^{*}, \\
& \quad k=\left(k_{1}, \ldots, k_{q}\right) \in \mathbb{N}^{q} .
\end{aligned}
$$

As a consequence, for $k \neq n$, only the terms with $p=1$ contribute to the linear part:

$$
\begin{aligned}
\operatorname{Tad}_{\mathscr{D}+\tilde{A}}(d-k) & =\operatorname{Lin}_{A}\left(f\left|\mathscr{D}_{A}\right|^{-(d-k)}\right) \\
& =\sum_{r=0}^{k-1} \operatorname{Res}_{s=d-k} h(s, r, 1) \operatorname{Tr}\left(\varepsilon^{r}\left(\operatorname{Lin}_{A}(Y)\right)|\mathscr{D}|^{-s}\right) .
\end{aligned}
$$


We check that for any $N \in \mathbb{N}^{*}$,

$$
\operatorname{Lin}_{A}(Y) \sim \sum_{l=0}^{N-1} \Gamma_{1}^{l}(\tilde{A} \mathcal{D}+\mathscr{D} \tilde{A}) \mathcal{D}^{-2(l+1)} \operatorname{mod~OP}{ }^{-N-1}
$$

Since $\Gamma_{1}^{l}(\tilde{A} \mathscr{D}+\mathscr{D} \tilde{A})=\frac{(-1)^{l}}{l+1} \nabla^{l}(\tilde{A} \mathscr{D}+\mathscr{D} \tilde{A})=\frac{(-1)^{l}}{l+1}\left\{\nabla^{l}(\tilde{A}), \mathscr{D}\right\}$, we get, assuming the dimension spectrum to be simple,

$$
\begin{aligned}
\operatorname{Tad}_{\mathscr{D}}+\tilde{A}^{(d-k)} & \\
= & \sum_{r=0}^{k-1} \operatorname{Res}_{s=d-k} h(s, r, p) \operatorname{Tr}\left(\varepsilon^{r}\left(\operatorname{Lin}_{A}(Y)\right)|\mathcal{D}|^{-s}\right) \\
& =\sum_{r=0}^{k-1} h(n-k, r, 1) \sum_{l=0}^{k-1-r} \frac{(-1)^{l}}{l+1} \operatorname{Res}_{s=d-k} \operatorname{Tr}\left(\varepsilon^{r}\left(\left\{\nabla^{l}(\tilde{A}), \mathscr{D}\right\}\right)|\mathcal{D}|^{-s-2(l+1)}\right) \\
& =2 \sum_{r=0}^{k-1} h(d-k, r, 1) \sum_{l=0}^{k-1-r} \frac{(-1)^{l}}{l+1} f \nabla^{r+l}(\tilde{A}) \mathscr{D}|\mathcal{D}|^{-(d-k+2(r+l))-2} \\
& =-(n-k) f \tilde{A} \mathscr{D}|\mathscr{D}|^{-(d-k)-2}
\end{aligned}
$$

because in the last sum it remains only the case $r+l=0$, so $r=l=0$.

Formula (9) is a direct application of [19], Lemma4.5. The link between $\operatorname{Tad}_{\mathscr{D}+\tilde{A}}$ and $\operatorname{Tad}_{\mathscr{D}+A}$ follows from $J \mathscr{D}=\epsilon \mathscr{D} J$ and Lemma 3.2. [26].

It is known that the noncommutative torus gives rise to a real spectra triple [11],

Corollary 3.6. There are no tadpoles in the noncommutative torus.

Proof. This follows from Proposition 3.5 and [19], Lemma 5.6.

Note that this is independent of a Diophantine condition.

Corollary 3.7. In a real spectral triple $(\mathcal{A}, H, \mathscr{D})$, if $A=A^{*} \in \Omega_{\mathscr{D}}^{1}(\mathcal{A})$ is such that $\tilde{A}=0$, then $\operatorname{Tad}_{D+A}(k)=0$ for any $k \in \mathbb{Z}, k \leq d$.

The vanishing tadpole of order 0 has the following equivalence (see [16])

$$
f A D^{-1}=0 \text { for all } A \in \Omega_{\mathscr{D}}^{1}(\mathcal{A}) \Longleftrightarrow f a b=f a \alpha(b) \text { for all } a, b \in \mathcal{A} \text {, }
$$

where $\alpha(b):=\mathscr{D} b \mathscr{D}^{-1}$.

We finish this section with a remark on the value of the zeta function at zero when the spectral triple is 2-dimensional. 
Lemma 3.8. In any spectral triple of dimension 2 (commutative or not) with vanishing tadpoles of order zero (i.e., (10) is satisfied) we have $\zeta_{D+A}(0)=\zeta_{D}(0)$ for any 1 form $A$.

Proof. Let $a_{1}, a_{2}, b_{1}, b_{2} \in \mathcal{A}$. Then, with $A_{1}=a_{1}\left[\mathfrak{D}, b_{1}\right]$,

$f A_{1} \mathscr{D}^{-1} a_{2}\left[\mathscr{D}, b_{2}\right] \mathscr{D}^{-1}=f A_{1}\left[\mathscr{D}^{-1}, a_{2}\right]\left[\mathscr{D}, b_{2}\right] D^{-1}+f A_{1} a_{2} \mathscr{D}^{-1}\left[\mathscr{D}, b_{2}\right] \mathscr{D}^{-1}$.

The first term is zero since the integrand is in $\mathrm{OP}^{-3}$, while the second term is equal to $f\left(a_{1} \alpha\left(b_{1} a_{2}\right)-a_{1} b_{1} \alpha\left(a_{2}\right)\right)\left(\alpha\left(b_{2}\right)-b_{2}\right)$, so is zero using $\alpha(x) \alpha(y)=\alpha(x y)$, $f x y=f x \alpha(y)$ by (10) and the fact that $f$ is a trace. Thus $f\left(A D^{-1}\right)^{2}=0$, and the result follows.

\section{Commutative spectral triples}

\subsection{Commutative geometry}

Definition 4.1. Consider a commutative spectral triple given by a compact Riemannian spin manifold $M$ of dimension $d$ without boundary and its Dirac operator $\mathscr{D}$ associated to the Levi-Civita connection. This means $\left(\mathcal{A}:=C^{\infty}(M), \mathscr{H}:=\right.$ $\left.L^{2}(M, S), D\right)$, where $S$ is the spinor bundle over $M$. This triple is real since, due to the existence of a spin structure, the charge conjugation operator generates an anti-linear isometry $J$ on $\mathscr{H}$ such that

$$
\mathrm{JaJ}^{-1}=a^{*} \quad \text { for all } a \in \mathcal{A},
$$

and when $d$ is even, the grading is given by the chirality matrix

$$
\chi_{M}:=(-i)^{d / 2} \gamma^{1} \gamma^{2} \ldots \gamma^{d} .
$$

Such a triple is said to be a commutative geometry (see [14] and [15] for the role of $J$ in the nuance between spin and $\operatorname{spin}^{c}$ manifold).

Since $J a J^{-1}=a^{*}$ for $a \in \mathcal{A}$, we get that in a commutative geometry

$$
J A J^{-1}=-\epsilon A^{*} \quad \text { for all } A \in \Omega_{\mathscr{D}}^{1}(\mathcal{A}) .
$$

As the following remark shows, being a commutative geometry is more than just having a commutative algebra:

Remark 4.2. Note that $\tilde{A}=0$ for all $A=A^{*} \in \Omega_{\mathscr{D}}^{1}$, when $\mathcal{A}$ is commutative and $J a J^{-1}=a^{*}$ for all $a \in \mathcal{A}$; see (12). Thus one can only use $\mathscr{D}_{A}=\mathscr{D}+A$.

But we can have $\mathcal{A}$ commutative and $J_{a} J^{-1} \neq a^{*}$ [14], [38]: 
Let $\mathcal{A}_{1}=\mathbb{C} \oplus \mathbb{C}$ represented on $\mathscr{H}_{1}=\mathbb{C}^{3}$ with, for some complex number $m \neq 0$,

$$
\begin{gathered}
\pi_{1}(a):=\left(\begin{array}{ccc}
b_{1} & 0 & 0 \\
0 & b_{1} & 0 \\
0 & 0 & b_{2}
\end{array}\right) \quad \text { for } a=\left(b_{1}, b_{2}\right) \in \mathcal{A}, \\
\mathscr{D}_{1}:=\left(\begin{array}{ccc}
0 & m & m \\
\bar{m} & 0 & 0 \\
\bar{m} & 0 & 0
\end{array}\right), \quad \chi_{1}:=\left(\begin{array}{rrr}
1 & 0 & 0 \\
0 & -1 & 0 \\
0 & 0 & -1
\end{array}\right), \quad J_{1}:=\left(\begin{array}{lll}
1 & 0 & 0 \\
0 & 0 & 1 \\
0 & 1 & 0
\end{array}\right) \circ \mathrm{cc},
\end{gathered}
$$

where cc is the complex conjugation. Then $\left(\mathcal{A}_{1}, \mathscr{H}_{1}, \mathscr{D}_{1}\right)$ is a commutative real spectral triple of dimension $d=0$ with non-zero 1 -forms and such that $J_{1} \pi_{1}(a) J_{1}^{-1}=$ $\pi_{1}\left(a^{*}\right)$ only if $a=\left(b_{1}, b_{1}\right)$.

Take a commutative geometry $\left(\mathcal{A}_{2}=C^{\infty}(M), \mathscr{H}=L^{2}(M, S), \mathscr{D}_{2}, \chi_{2}, J_{2}\right)$ introduced in Definition 4.1, where $d=\operatorname{dim} M$ is even, and then the tensor product of the two spectral triples, namely $\mathcal{A}=\mathcal{A}_{1} \otimes \mathcal{A}_{2}, \mathscr{H}=\mathscr{H}_{1} \otimes \mathscr{H}_{2}, \mathcal{D}=\mathscr{D}_{1} \otimes \chi_{2}+$ $1 \otimes D_{2}, \chi=\chi_{1} \otimes \chi_{2}$, and $J$ is either $\chi_{1} J_{1} \otimes J_{2}$ when $d \in\{2,6\} \bmod 8$, or $J_{1} \otimes J_{2}$ otherwise; see [14], [47].

Then $(\mathcal{A}, \mathscr{H}, \mathscr{D})$ is a real commutative triple of dimension $d$ such that $\tilde{A} \neq 0$ for some selfadjoint 1-form $A$, so is not exactly like in Definition 4.1.

4.2. No tadpoles. The appearance of tadpoles never occurs in commutative geometries, as quoted in [17], p. 212, Lemma 1.149, for the dimension $d=4$. This fact means that a given geometry $(\mathcal{A}, \mathscr{H}, \mathscr{D})$ is a critical point for the spectral action (1) [17], p. 210.

Theorem 4.3. There are no tadpoles on a commutative geometry. Namely, for any 1-form $A=A^{*} \in \Omega_{\mathscr{D}}^{1}(\mathcal{A}), \operatorname{Tad}_{\mathscr{D}+A}(d-k)=0$, for any $k \in \mathbb{N}$.

Proof. Since $\tilde{A}=0$ when $A=A^{*}$ by (12), the result follows from Corollary 3.7.

There are similar results in the following

Lemma 4.4. Under same hypothesis, for any $k, l \in \mathbb{N}$, the following hold:

(i) $f A D^{-k}=-\epsilon^{k+1} f A D^{-k}$.

(ii) $f \chi A D^{-k}=-\epsilon^{k+1} f \chi A D^{-k}$.

(iii) $f A^{l}|\mathcal{D}|^{-k}=(-\epsilon)^{l} f A^{l}|\mathcal{D}|^{-k}$.

(iv) $f \chi A^{l}|\mathcal{D}|^{-k}=(-\epsilon)^{l} f \chi A^{l}|\mathcal{D}|^{-k}$. 
Proof. To show (i), consider

$$
\begin{aligned}
f A D^{-k} & =\overline{f J A D^{-k} J^{-1}} \\
& =\overline{f J A J^{-1}\left(\epsilon^{k} \mathscr{D}^{-k}\right)} \\
& =-\epsilon^{k+1} \overline{f A^{*} \mathscr{D}^{-k}}=-\epsilon^{k+1} f \mathscr{D}^{-k} A=-\epsilon^{k+1} f A D^{-k} .
\end{aligned}
$$

The same argument gives the other equalities using $\chi A=-A \chi$ and $\chi|\mathscr{D}|=|\mathscr{D}| \chi$.

Lemma 4.5. For any 1-form $A, f\left(A D^{-1}\right)^{k}=0$ when $k \in \mathbb{N}$ is odd.

Proof. We have

$$
\begin{aligned}
f\left(A D^{-1}\right)^{k} & =\overline{f J\left(A D^{-1}\right)^{k} J^{-1}} \\
& =\overline{f\left(J A J^{-1} J D^{-1} J^{-1}\right)^{k}} \\
& =(-1)^{k} \epsilon^{2 k} \overline{f\left(A^{*} D^{-1}\right)^{k}}=(-1)^{k} f\left(A D^{-1}\right)^{k}
\end{aligned}
$$

(which shows again that $f A D^{-1}=0$ ).

4.3. Tadpole-like integrals. The goal of this section is to prove the following theorem, which extends Theorem 4.3 to tadpole-like integrals of the form $f B|D|^{-k}$ :

Theorem 4.6. Let $(\mathcal{A}, \mathcal{H}, \mathcal{D})$ be a commutative geometry of dimension $d$.

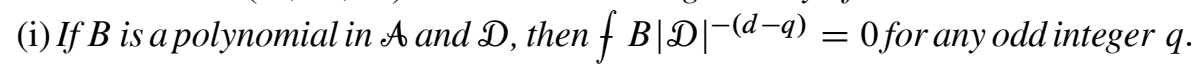

(ii) If $B$ is an element of the polynomial algebra generated by $\mathcal{A}$ and $[D, \mathcal{A}]$, then

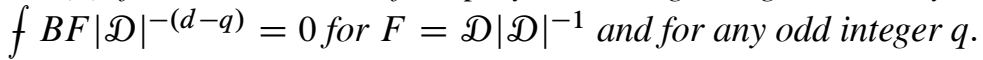

(iii) For any 1-form $A, f A|D|^{-q}=0, q \in \mathbb{N}$, in either of the following cases:

$d \neq 1 \bmod 8$ and $d \neq 5 \bmod 8$,

$d=1 \bmod 8$ or $d=5 \bmod 8$ and $q$ is even or $q \geq \frac{d+3}{2}$.

In order to prove that these integrals are zero despite the fact that the use of the operator $J$ in the trick (13) is not sufficient, we need to use the Wodzicki residue (see 
[51], [52]): in a chosen coordinate system and local trivialization $(x, \xi)$ of $T^{*} M$, this residue is

$$
\operatorname{wres}_{x}(X):=\int_{S_{x}^{*} M} \operatorname{Tr}\left(\sigma_{-d}^{X}(x, \xi)\right)|d \xi|\left|d x^{1} \wedge \cdots \wedge d x^{d}\right|,
$$

where $\sigma_{-d}^{X}(x, \xi)$ is the symbol of the classical pseudodifferential operator $X$ in the chosen coordinate frame $\left(x_{1}, \ldots, x_{d}\right)$, which is homogeneous of degree $-d:=$ $-\operatorname{dim}(M)$ and taken at point $(x, \xi) \in T^{*}(M)$, and $d \xi$ is the normalized restriction of the volume form to the unit sphere $S_{x}^{*} M \simeq \mathbb{S}^{d-1}$; so we assume $d \geq 2$ to get $S_{x}^{*} M$ connected.

This $\operatorname{wres}_{x}(X)$ appears to be a one-density not depending on the local representation of the symbol (see [52], [26]), so

$$
\operatorname{Wres}(X):=\int_{M} \operatorname{wres}_{x}(X)
$$

is well defined.

The noncommutative integral $f$ coincides with the Wodzicki residue, up to a scalar: since both $f$ and Wres are traces on the set of pseudodifferential operators, the uniqueness of the trace [52] gives the proportionality

$$
f X=c_{d} \operatorname{wres}(X)
$$

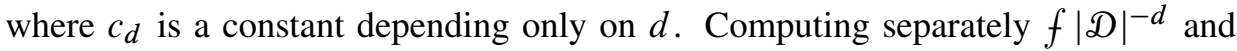
Wres $\left(|\mathscr{D}|^{-d}\right.$ ), we get $c_{d}>0$ (note that $f$ is not a positive functional, see Lemma 4.14).

Lemma 3.2 follows for instance from the fact that $\int_{M} \operatorname{wres}_{x}\left(X^{*}\right)=\overline{\int_{M} \operatorname{wres}_{x}(X)}$. Note that Wres is independent of the metric.

As noticed by Wodzicki, $f X$ is equal to -2 times the coefficient in $\log t$ of the asymptotics of $\operatorname{Tr}\left(X e^{-t \mathscr{D}^{2}}\right)$ as $t \rightarrow 0$. It is remarkable that this coefficient is independent of $\mathscr{D}$ and this gives a close relation between the $\zeta$ function and heat kernel expansion with Wres. Actually, by [28], Theorem 2.7,

$$
\operatorname{Tr}\left(X e^{-t \mathscr{D}^{2}}\right) \sim_{t \rightarrow 0^{+}} \sum_{k=0}^{\infty} a_{k} t^{(j-o r d(X)-d) / 2}+\sum_{k=0}^{\infty}\left(-a_{k}^{\prime} \log t+b_{k}\right) t^{k},
$$

so $f X=2 a_{0}^{\prime}$. Since, via Mellin transform,

$$
\operatorname{Tr}\left(X \mathscr{D}^{-2 s}\right)=\frac{1}{\Gamma(s)} \int_{0}^{\infty} t^{s-1} \operatorname{Tr}\left(X e^{-t \mathscr{D}^{2}}\right) d t,
$$

the non-zero coefficient $a_{k}^{\prime}, k \neq 0$, creates a pole of $\operatorname{Tr}\left(X D^{-2 s}\right)$ of order $k+2$ since $\int_{0}^{1} t^{s-1} \log (t)^{k}=\frac{(-1)^{k} k !}{s^{k+1}}$ and

$$
\Gamma(s)=\frac{1}{s}+\gamma+s g(s),
$$


where $\gamma$ is the Euler constant and the function $g$ is also holomorphic around zero.

We have $f 1=0$ and more generally, $\operatorname{Wres}(P)=0$ for all zero-order pseudodifferential projections [51].

For extension to log-polyhomogeneous pseudodifferential operators, see [39].

When $M$ has a boundary, some $a_{k}^{\prime}$ are non-zero, the dimension spectrum can be non simple (even if it is simple for the Dirac operator, see for instance [40].)

On a spectral triple $(\mathcal{A}, \mathscr{H}, \mathcal{D})$, the fact to change the product on $\mathcal{A}$ may or not affect the dimension spectrum: for instance, there is no change when one goes from the commutative torus to the noncommutative one (see [19]), while the dimension spectrum of $\mathrm{SU}_{q}$ (2), which is bounded from below, does not coincide with the dimension spectrum of the sphere $\mathbb{\$}^{3}$ corresponding to $q=1$; see [33], Corollary 4.10.

We first introduce few necessary notations. In the following we fix a local coordinate frame $\left(U,\left(x_{i}\right)_{1 \leq i \leq n}\right)$ which is normal at $x_{0} \in M$, and denote by $\sigma_{k}^{X}$ the $k$-homogeneous symbol of any classical pseudodifferential operator $X$ on $M$, in this local coordinate frame. We denote by $g$ the Riemannian metric on $M$ and by $g_{i j}:=[g]_{i j}$ its coordinates. The Dirac operator is locally of the form, compatible with (4),

$$
\mathscr{D}=-i \gamma\left(d x^{j}\right)\left(\partial_{x^{j}}+\omega_{j}(x)\right),
$$

where $\omega_{j}$ is the spin connection, $\gamma$ is the Clifford multiplication of 1-forms; see [26], p. 392. Here we make the choice of gauge given by $[h]:=\sqrt{[g]}$, which leads with $[\tilde{h}]:=[h]^{-1}$ to (see [26], Exercise 9.6)

$$
\omega_{i}=-\frac{1}{4}\left(\Gamma_{i j}^{k} g_{k l}-\partial_{x^{j}}\left(h_{j}^{\alpha}\right) \delta_{\alpha \beta} h_{l}^{\beta}\right) \gamma\left(d x^{j}\right) \gamma\left(d x^{l}\right), \quad \gamma\left(d x^{j}\right)=\tilde{h}^{j k} \gamma_{k},
$$

where $\gamma^{j}=\gamma_{j}$ are the selfadjoint constant $\gamma$ matrices satisfying $\left\{\gamma^{i}, \gamma^{j}\right\}=\delta^{i j}$.

Thus

$$
\sigma^{\mathscr{D}}(x, \xi)=\tilde{h}^{j k} \gamma_{k}\left(\xi_{j}-i \omega_{j}(x)\right) .
$$

We have chosen normal (or geodesic) coordinates around the base point $x_{0}$. Since

$$
\begin{gathered}
g_{i j}(x)=g_{i j}\left(x_{0}\right)+\frac{1}{3} R_{i j k l} x^{k} x^{l}+o\left(\|x\|^{3}\right), \\
g^{i j}(x)=g^{i j}\left(x_{0}\right)-\frac{1}{3} R^{i}{ }_{k}{ }^{j}{ }^{k} x^{k} x^{l}+o\left(\|x\|^{3}\right), \\
g_{i j}\left(x_{0}\right)=\delta_{i j}, \quad \Gamma_{i j}^{k}\left(x_{0}\right)=0,
\end{gathered}
$$

the matrices $h(x)$ and $h^{-1}(x)$ have no linear terms in $x$. Thus

$$
\omega_{i}\left(x_{0}\right)=0 .
$$

We could also have said that parallel translation of a basis of the cotangent bundle along the radial geodesics emanating from $x_{0}$ yields a trivialization (this is the radial gauge) such that $\omega_{i}\left(x_{0}\right)=0$. In particular, using product formula for symbols and 
the fact that in the decomposition $D=\mathscr{D}+P, P \in \mathrm{OP}^{-\infty}$, we get for $k \in \mathbb{N}$

$$
\begin{aligned}
\sigma_{1}^{\mathcal{D}}(x, \xi) & =\tilde{h}^{j k}(x) \gamma_{k} \xi_{j}=\gamma(\xi), & & \sigma_{1}^{\mathcal{D}}\left(x_{0}, \xi\right)=\gamma^{j} \xi_{j}, \\
\sigma_{0}^{\mathcal{D}}(x, \xi) & =-i \tilde{h}^{j k}(x) \gamma_{k} \omega_{j}(x), & & \sigma_{0}^{\mathcal{D}}\left(x_{0}, \xi\right)=0, \\
\partial_{x^{k}} \sigma_{1}^{\mathcal{D}}\left(x_{0}, \xi\right) & =0, & & \\
\sigma_{-1}^{D^{-1}}(x, \xi) & =\tilde{h}^{j k}(x) \gamma_{j} \xi_{k}\|\xi\|_{x}^{-2}, & & \|\xi\|_{x}^{2}:=g^{j k}(x) \xi_{j} \xi_{k}, \\
\partial_{x^{k}} \sigma_{-1}^{D^{-1}}\left(x_{0}, \xi\right) & =0 . & &
\end{aligned}
$$

We will use freely the fact that the symbol of a 1 -form $A$ can be written as

$$
\sigma^{A}(x, \xi)=\sigma_{0}^{A}(x)=-i a_{k}(x) \gamma^{k}
$$

with $a_{k}(x) \in i \mathbb{R}$ when $A=A^{*}$.

When $d$ is even (so $\epsilon=1$ ), observe that for $k=l, A_{i}=a_{i}\left[D, b_{i}\right]$ and $a=$ $\prod_{i=1}^{k} a_{i}$, by [18], p. 231 (actually, $\chi$ is missing), [43] or [26], p. 479, when $k=d$ ( $M$ is supposed to be oriented),

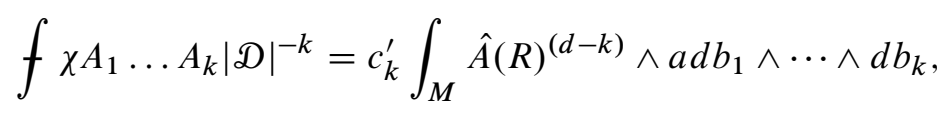

where $\hat{A}(R)$ is the $\hat{A}$-genus associated to the Riemannian curvature $R$. It follows from $\hat{A}(R) \in \bigoplus_{j \in \mathbb{N}} \Omega^{4 j}(M, \mathbb{R})$ that $f \chi A^{k}|D|^{-k}$ can be non-zero only when $k=d-4 j$. For instance in dimension $d=2$, for $j=0$,

$$
\begin{aligned}
\sigma_{-2}^{\chi A_{1} A_{2} \mathscr{D}^{-2}}(x, \xi) & =\sigma_{0}^{\chi A_{1} A_{2}}(x) \sigma_{-2}^{D^{-2}}(x, \xi) \\
& =-a_{1}(x) a_{2}(x) \chi g^{j k}(x) \gamma_{j} \gamma_{k} \frac{1}{g^{l m}(x) \xi_{l} \xi_{m}}
\end{aligned}
$$

Thus $\operatorname{wres}_{x}\left(\chi A_{1} A_{2} D^{-2}\right)=-2 a_{1}(x) a_{2}(x) \sqrt{\operatorname{det} g_{x}} \operatorname{Tr}\left(\chi \gamma^{j} \gamma^{k}\right)$, so if $v_{g}$ is the Riemannian density, we have

$$
f \chi A_{1} A_{2} D^{-2}=-2 c_{d} \operatorname{Tr}\left(\chi \gamma^{j} \gamma^{k}\right) \int_{M} a_{1} a_{2} v_{g} .
$$

Actually, this last equality is nothing else than Wodzicki-Connes' trace theorem, see [26], Section 7.6, and this is equal to $c_{d}^{\prime} \int_{M} a_{1} a_{2} d b_{1} \wedge d b_{2}$ as claimed above.

We now introduce a few subspaces of the pseudodifferential operators space $\Psi(M)$. Let

$$
\begin{array}{ll}
\mathcal{B}_{\mathrm{e}}:=\left\{P \in \Psi(M): \sigma_{j}^{P} \in E_{j} \text { for all } j \in \mathbb{Z}\right\}, & \text { e for even, } \\
\mathcal{B}_{\mathrm{o}}:=\left\{P \in \Psi(M): \sigma_{j}^{P} \in O_{j} \text { for all } j \in \mathbb{Z}\right\}, & \text { o for odd, }
\end{array}
$$


such that, for $m=2^{[d / 2]}$,

$$
\begin{gathered}
E_{j}:=\left\{f \in C^{\infty}\left(U \times \mathbb{R}^{d} \backslash\{0\}, \mathcal{M}_{m}(\mathbb{C})\right): f(x, \xi)=\sum_{i \in I} \frac{\xi^{\beta^{i}}}{\|\xi\|_{x}^{2 k_{i}}} h_{i}(x), I \neq \emptyset,\right. \\
\left.k_{i} \in \mathbb{N}, \beta^{i} \in \mathbb{N}^{d},\left|\beta^{i}\right|-2 k_{i}=j, h_{i} \in C^{\infty}\left(U, \mathcal{M}_{m}(\mathbb{C})\right)\right\}, \\
O_{j}:=\left\{f \in C^{\infty}\left(U \times \mathbb{R}^{d} \backslash\{0\}, \mathcal{M}_{m}(\mathbb{C})\right): f(x, \xi)=\sum_{i \in I} \frac{\xi^{\beta^{i}}}{\|\xi\|_{x}^{2 k_{i}+1}} h_{i}(x), I \neq \emptyset,\right. \\
\left.k_{i} \in \mathbb{N}, \beta^{i} \in \mathbb{N}^{d},\left|\beta^{i}\right|-\left(2 k_{i}+1\right)=j, h_{i} \in C^{\infty}\left(U, \mathcal{M}_{m}(\mathbb{C})\right)\right\} .
\end{gathered}
$$

The space $\mathscr{B}_{\mathrm{e}}$ and $\mathscr{B}_{\mathrm{o}}$ capture the information associated to the even or odd character of the polyhomogeneous expansion of the pseudodifferential operators symbols.

We also want to pay attention to the real or purely imaginary nature (independently of the appearance of gamma matrices) of homogeneous symbols of a given pseudodifferential operator. For this reason, we define

$$
\lessdot:=\left\{P \in \Psi^{p}(M): \sigma_{p-j}^{P} \in I_{j} \text { for all } j \in \mathbb{N}\right\},
$$

where $I_{k}=I_{e}$ if $k$ is even and $I_{k}=I_{o}$ if $k$ is odd, with

$$
\begin{aligned}
& I_{e}:=\left\{f \in C^{\infty}\left(U \times \mathbb{R}^{n}, \mathcal{M}_{m}(\mathbb{C})\right): f=\gamma_{k_{1}} \ldots \gamma_{k_{q}} h(x, \xi), h \text { real valued }\right\} \\
& I_{o}:=\left\{f \in C^{\infty}\left(U \times \mathbb{R}^{n}, \mathcal{M}_{m}(\mathbb{C})\right): f=i \gamma_{k_{1}} \ldots \gamma_{k_{q}} h(x, \xi), h \text { real valued }\right\}
\end{aligned}
$$

These spaces satisfy the following technical lemmas, which are proven in Appendix A.3:

Lemma 4.7. For any $j, j^{\prime} \in \mathbb{Z}$ and $\alpha \in \mathbb{N}^{d}$, the following hold:

(i) $E_{j} E_{j^{\prime}} \subseteq E_{j+j^{\prime}}$ and $\partial_{\xi}^{\alpha} E_{j} \subseteq E_{j-|\alpha|}, \partial_{x}^{\alpha} E_{j} \subseteq E_{j}$

(ii) $O_{j} O_{j^{\prime}} \subseteq E_{j+j^{\prime}}$ and $\partial_{\xi}^{\alpha} O_{j} \subseteq O_{j-|\alpha|}, \partial_{x}^{\alpha} O_{j} \subseteq O_{j}$.

(iii) $O_{j} E_{j^{\prime}}$ and $E_{j^{\prime}} O_{j}$ are included in $O_{j+j^{\prime}}$.

(iv) $\mathcal{B}_{\mathrm{e}}$ is a subalgebra of $\Psi(M)$.

(v) $\mathcal{B}_{\mathrm{e}} \mathcal{B}_{\mathrm{e}}, \mathcal{B}_{\mathrm{o}} \mathcal{B}_{\mathrm{o}}$ are included in $\mathcal{B}_{\mathrm{e}}$, and $\mathcal{B}_{\mathrm{e}} \mathcal{B}_{\mathrm{o}}, \mathcal{B}_{\mathrm{o}} \mathcal{B}_{\mathrm{e}}$ are included in $\mathcal{B}_{\mathrm{o}}$.

Lemma 4.8. (i) Let $P \in \mathscr{B}_{\mathrm{e}}$ (resp. $\mathscr{B}_{\mathrm{o}}$ ) be an elliptic classical pseudodifferential operator in $\Psi^{p}(M)$ with $\sigma_{p}^{P}(x, \xi)=\|\xi\|_{x}^{p}, p \in \mathbb{N}$. Then any parametrix $P^{-1}$ of $P$ is in $\mathcal{B}_{\mathrm{e}}$ (resp. $\mathcal{B}_{\mathrm{o}}$ ).

(ii) For any $k \in \mathbb{Z}, \mathscr{D}^{k} \in \mathscr{B}_{\mathrm{e}}$ and when $k$ is odd, $|\mathscr{D}|^{k} \in \mathscr{B}_{\mathrm{o}}$.

(iii) If $d$ is odd, then $f P=0$ for any $P \in \mathcal{B}_{\mathrm{e}}$.

(iv) If $d$ is even, then $f P=0$ for any $P \in \mathscr{B}_{0}$.

(v) For any pseudodifferential operator $P \in \Psi_{1}(\mathcal{A})$ (see Appendix A.1),

when $d$ is odd, then $f P=0$,

when $d$ is even, then $f P|D|^{-1}=0$. 
Lemma 4.9. (i) $C$ is a subalgebra of $\Psi(M)$.

(ii) If $P \in \mathcal{C}$ is elliptic then $P^{-1} \in \mathcal{C}$.

(iii) $\mathscr{D}^{k} \in \mathcal{C}$ and $|\mathscr{D}|^{k} \in \mathcal{C}$ for any $k \in \mathbb{Z}$.

We can now prove the main result of this section:

Proof of Theorem 4.6. (i) This is a direct consequence of Lemma 4.8 (v).

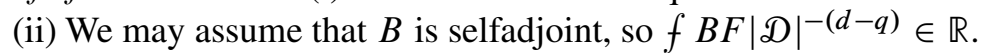

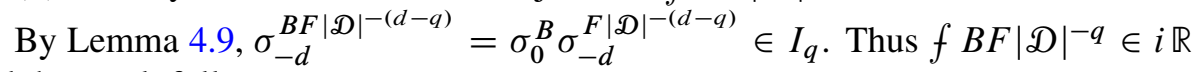
and the result follows.

(iii) In the case $d \neq 1 \bmod 8$ and $d \neq 5 \bmod 8$, the result follows from the fact that $\epsilon=1$.

The case $d$ even and $q$ odd or $d$ odd and $q$ even is done by Lemma $4.8(\mathrm{v})$.

Suppose that $d$ is even and $q$ is even. In this case we look at the information given by the gamma matrices. If $q=2 k$, with a recurrence and the symbol product formula, we see that $\sigma_{2 k-j}^{D^{2 k}}$ and all its derivatives are linear combinations of terms of the form $f(x, \xi) \otimes \gamma^{j_{1}} \ldots \gamma^{j_{i}}$, where $i$ is even and less than $2 j$ (with the convention $\gamma^{j_{1}} \ldots \gamma^{j_{i}}=1$ if $\left.i=0\right)$. We call this property $\left(P_{j}\right)$. The parametrix equation $\mathscr{D}^{2 k} \mathscr{D}^{-2 k}=1$ entails that $\sigma_{-2 k}^{D^{-2 k}}=\left(\sigma_{2 k}^{D^{2 k}}\right)^{-1}$ and for any $j \geq 1$,

$$
\begin{aligned}
& \sigma_{-2 k-j}^{D^{-2 k}}=-\sigma_{-2 k}^{\mathcal{D}^{-2 k}}\left(\sum_{r=\max \{j-2 k, 0\}}^{j-1} \sigma_{2 k-(j-r)}^{\mathfrak{D}^{2 k}} \sigma_{-2 k-r}^{\mathfrak{D}^{-2 k}}\right. \\
& \left.+\sum_{1 \leq|\alpha| \leq 2 k} \sum_{r=\max \{j-2 k, 0\}}^{j-|\alpha|} \frac{(-i)^{|\alpha|}}{\alpha !} \partial_{\xi}^{\alpha} \sigma_{2 k-(j-|\alpha|-r)}^{D^{2 k}} \partial_{x}^{\alpha} \sigma_{-2 k-r}^{D^{-2 k}}\right) .
\end{aligned}
$$

Note that $\sigma_{-2 k}^{D^{-2 k}}$ satisfies $\left(P_{0}\right)$. By recurrence, this formula shows that $\sigma_{-2 k-j}^{D^{-2 k}}$ satisfies $\left(P_{j}\right)$ for any $j \in \mathbb{N}$. In particular, $\sigma_{-d}^{D^{-2 k}}$ satisfies $\left(P_{-2 k+d}\right)$ and the result then follows from (23), and the fact that the product of an odd number (different from the dimension) of gamma matrices is traceless.

Suppose now that $d$ is odd, $q$ is odd and $d \geq q$. In that situation, any odd number of gamma matrices $\gamma^{i_{1}} \ldots \gamma^{i_{r}}$ is traceless when $r<d$.

Using the parametrix equation $|\mathscr{D}|^{-q}|\mathscr{D}|^{-q}=\mathscr{D}^{-2 q}$, once can check that $\sigma_{-q}^{|\mathscr{D}|^{-q}}(x, \xi)=\|\xi\|_{x}^{-q}$ and for any $j \in \mathbb{N}^{*}$,

$$
\begin{aligned}
\sigma_{-q-j}^{|\mathscr{D}|^{-q}}= & \frac{1}{2\|\xi\|_{x}^{-q}}\left(\sigma_{-2 q-j}^{\mathcal{D}^{-2 q}}-\sum_{0<k<j} \sigma_{-q-j+k}^{|\mathscr{D}|^{-q}} \sigma_{-q-k}^{|\mathscr{D}|^{-q}}\right. \\
& \left.+\sum_{0<|\alpha| \leq j} \sum_{k=0}^{j-|\alpha|} i^{|\alpha|} \frac{(-1)^{|\alpha|}}{\alpha !} \partial_{\xi}^{\alpha} \sigma_{-q-j+|\alpha|+k}^{|\mathscr{D}|^{-q}} \partial_{x}^{\alpha} \sigma_{-q-k}^{|\mathscr{D}|^{-q}}\right)
\end{aligned}
$$


We saw that each $\sigma_{-2 q-j}^{|\mathscr{D}|^{-2 q}}$ satisfies $\left(P_{j}\right)$, that is to say, is a linear combination of terms of the form $f(x, \xi) \otimes \gamma^{j_{1}} \ldots \gamma^{j_{i}}$, where $i$ is even and less than $2 j$. Again, a straightforward induction argument shows that $\sigma_{-q-j}^{|\mathscr{D}|^{-q}}$ satisfies $\left(P_{j}\right)$ for any $j \in \mathbb{N}$. In particular, $\sigma_{-d}\left(A|D|^{-q}\right)$ is a linear combination of terms of the form $f(x, \xi) \otimes$ $\gamma^{j_{1}} \ldots \gamma^{j_{r}}$ where $r \leq 2(d-q)+1$ is odd. This yields the result.

Finally, we note that the equality $f A D^{-d+1}=0$, a consequence of Theorem 4.6, is also a consequence of the fact that $\sigma_{-d}^{\mathcal{D}^{-d+1}}\left(x_{0}, \xi\right)=0$ :

Lemma 4.10. For all $k \in \mathbb{N}^{*}$, we have $\sigma_{k-1}^{D^{k}}\left(x_{0}, \xi\right)=\sigma_{-k-1}^{D^{-k}}\left(x_{0}, \xi\right)=0$.

Proof. We already know that $\sigma_{0}^{D}\left(x_{0}, \xi\right)=0$, see (19). We proceed by recurrence, assuming $\sigma_{k-1}^{D^{k}}\left(x_{0}, \xi\right)=0$ for $k=1, \ldots, n$. Then $\sigma_{n}^{\mathscr{D}^{n+1}}=\sigma_{n}^{\mathscr{D}^{n}} \sigma_{0}^{\mathscr{D}}+\sigma_{n-1}^{\mathscr{D}^{n}} \sigma_{1}^{\mathscr{D}}-$ $i \partial_{\xi_{k}} \sigma_{n}^{D^{n}} \partial_{x^{k}} \sigma_{1}^{\mathcal{D}}$, thus by (19) and (20), $\sigma_{n}^{\mathscr{D}^{n+1}}\left(x_{0}, \xi\right)=0$.

Since $\mathscr{D} \mathscr{D}^{-1}=1$ yields $\sigma_{-2}^{\mathcal{D}^{-1}}\left(x_{0}, \xi\right)=-\left(\sigma_{-1}^{\mathcal{D}^{-1}} \sigma_{0}^{\mathcal{D}}\right)\left(x_{0}, \xi\right)=0$, we assume $\sigma_{-k-1}^{\mathcal{D}^{-k}}\left(x_{0}, \xi\right)=0$ for $k=1, \ldots n$. Then $\sigma_{-n-2}^{\mathcal{D}^{-n-1}}=\sigma_{-n}^{\mathcal{D}^{-n}} \sigma_{-2}^{\mathcal{D}^{-1}}+\sigma_{-n-1}^{\mathcal{D}^{-n}} \sigma_{-1}^{\mathcal{D}^{-1}}-$ $i \partial_{\xi_{k}} \sigma_{-n}^{D^{-n}} \partial_{x^{k}} \sigma_{-1}^{D^{-1}}$. Using (22) and recurrence hypothesis, $\sigma_{-n-2}^{D^{-n-1}}\left(x_{0}, \xi\right)=0$.

4.4. Remarks on nonlinear terms. We conclude this section with a few remarks on terms in the spectral action expansion (1) that, in the case of commutative geometries, are nonlinear in the perturbation $A$.

Remark 4.11. The function $\zeta_{X}(s):=\operatorname{Tr}\left(|X|^{-s}\right)$ is regular at point 0 when $X$ is an elliptic selfadjoint differential operator of order one (see [24]):

One checks that $\zeta_{X}(s)=\frac{1}{\Gamma(s)} \int_{0}^{\infty} t^{s-1} \operatorname{Tr}\left(e^{-t|X|}\right) d t$ for $\operatorname{Re}(s)>d$. Because of the asymptotic expansion

$$
\operatorname{Tr}\left(e^{-t|X|}\right)=t^{-d} \sum_{n=0}^{N} t^{n} a_{n}[X]+\mathcal{O}\left(t^{N+1-d}\right)
$$

and meromorphic extension to the whole complex plane, $\operatorname{Res}_{s=d-n} \zeta_{X}(s)=\frac{a_{n}[X]}{\Gamma(d-n)}$. In particular, $\zeta_{X}(s)=\Gamma(s)^{-1}\left(\frac{a_{d}[X]}{s}+f(s)\right)$, where $f$ is holomorphic around $s=0$. By (16) we get that $\zeta_{X}(s)$ is regular around zero and $\zeta_{X}(0)=a_{d}[X]$ if $d$ is even and $\zeta_{X}(0)=0$ if $d$ is odd.

Corollary 4.12. $\zeta_{D+A}(0)=\zeta_{\mathscr{D}}(0)=0$ when $d=\operatorname{dim}(M)$ is odd.

When $d$ is even, $\zeta_{\mathscr{D}+A}(0)-\zeta_{\mathscr{D}}(0)=\sum_{k=1}^{d / 2} \frac{1}{2 k} f\left(A D^{-1}\right)^{2 k}$.

Proof. The result follows from (2) and Lemma 4.5. 
A proof of (2) also follows from $\sigma^{\log \left(1+A D^{-1}\right)} \sim \sum_{k=1}^{\infty} \frac{(-1)^{k}}{k} \sigma^{\left(A D^{-1}\right)^{k}}$ with $\log (X):=\left.\frac{\partial}{\partial z}\right|_{z=0} X^{z}$. Thus Wres $\left.\left(\log \left(1+A D^{-1}\right)\right)=\sum_{k=1}^{d} \frac{(-1)^{k}}{k} \operatorname{Wres}\left(A D^{-1}\right)^{k}\right)$ since $\left(A D^{-1}\right)^{k}$ has zero Wodzicki residue if $k>d$ and moreover $\zeta_{\mathscr{D}+A}(0)=$ $-\mathrm{Wres}(\log (\mathscr{D}+A))$. Actually, the important point is that $\operatorname{det}(X):=e^{\mathrm{Wres}(\log (X))}$ is multiplicative (see [41].) Moreover, such determinant is different from the $\zeta$ determinant $e^{-\zeta_{X}^{\prime}(0)}$ used for instance by Hawking [30] in his regularization via the partition function which suffers from conformal anomalies.

The fact that in the asymptotic expansion (25) of the heat kernel the term $a_{2}[\mathscr{D}+A]$ does depend only on the scalar curvature, so is independent of $A$, is reflected in Lemma 3.8 when the dimension is equal to 2.

Note that $\zeta_{\mathscr{D}+A}(0)-\zeta_{\mathscr{D}}(0)$ is usually non-zero: consider for instance the flat 4-torus and as a generic selfadjoint 1 -form $A$, take

$$
A:=\phi \in\left[0,2 \pi\left[^{4} \mapsto-i \gamma^{\alpha} \sum_{l \in \mathbb{Z}^{4}} a_{\alpha, l} e^{i l^{k} \phi_{k}},\right.\right.
$$

where $a_{\alpha, l}$ is in the Schwartz space $S\left(Z^{4}\right)$ and $a_{\alpha, l}=-\overline{a_{\alpha,-l}}$. We have by [19], Lemma 6.12 (with $c=\frac{8 \pi^{2}}{3},|l|^{2}=\sum_{k} l^{k^{2}}$ and $\Theta=0$ ),

$$
\zeta_{\mathscr{D}+A}(0)-\zeta_{\mathscr{D}}(0)=f\left(A \mathscr{D}^{-1}\right)^{2}=c \sum_{l \in \mathbb{Z}^{4}} a_{\alpha_{1}, l} a_{\alpha_{2},-l}\left(l^{\alpha_{1}} l^{\alpha_{2}}-\delta^{\alpha_{1} \alpha_{2}}|l|^{2}\right)
$$

since $f\left(A D^{-1}\right)^{4}=0$.

This last equality suggests that Lemma 4.5 can be extended:

Proposition 4.13. For any 1-form $A, f\left(A D^{-1}\right)^{d}=0$ if $d=\operatorname{dim}(M)$.

Proof. As in the proof of Lemma 3.8, $\mathscr{D}^{-1}$ commutes with the element in the algebra as the integrand is in $\mathrm{OP}^{-d}$. So for a family of $a_{i}, b_{i} \in \mathcal{A}$ and using $a:=\prod_{i=1}^{d} a_{i}$,

$$
f \prod_{i=1}^{d}\left(a_{i}\left[\mathcal{D}, b_{i}\right] D^{-1}\right)=f\left(\prod_{i=1}^{d} a_{i}\right) \prod_{i=1}^{d}\left(\left[\mathcal{D}, b_{i}\right] \mathcal{D}^{-1}\right)=f a \prod_{i=1}^{d}\left(\alpha\left(b_{i}\right)-b_{i}\right) \text {. }
$$

We obtain, since $\alpha\left(b_{i}\right)-b_{i} \in \mathrm{OP}^{-1}$,

$$
\sigma_{-d}^{a \prod_{i=1}^{d} \alpha\left(b_{i}\right)-b_{i}}=a \prod_{i=1}^{d} \sigma_{-1}^{\alpha\left(b_{i}\right)-b_{i}}=a \prod_{i=1}^{d} \sigma_{-1}^{\alpha\left(b_{i}\right)}
$$

Further, $\sigma_{-1}^{\mathscr{D} b_{i}} \mathscr{D}^{-1}\left(x_{0}, \xi\right)=0$ : we already know by Lemma 4.10 that $\sigma_{-2}^{\mathscr{D}^{-1}}\left(x_{0}, \xi\right)=$ 0 , by (21) that $\partial_{x^{k}} \sigma_{-1}^{D^{-1}}\left(x_{0}, \xi\right)=0$ for all $k$, and $\sigma_{0}^{D b_{i}}\left(x_{0}, \xi\right)=b_{i}\left(x_{0}\right) \sigma_{0}^{\mathscr{D}}\left(x_{0}, \xi\right)=$ 0 giving the claim and the result. 
This proposition does not survive in noncommutative spectral triples, see for instance [33], Table 1.

Note that for a 1 -form $A, f A^{d} D^{-d} \neq f\left(A D^{-1}\right)^{-d}=0$ : in dimension $d=2$, as in (24),

$$
f A^{2} D^{-2}=-2 c_{d} \operatorname{Tr}\left(\gamma^{k} \gamma^{l}\right) \int_{M} a_{k} a_{l} v_{g} .
$$

It is known (see [17], p. 214, Proposition 1.153) that the $d-2$ term (for $d=4$ )

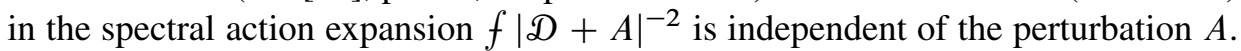
This is why the Einstein-Hilbert action $S(\mathscr{D})=f|\mathscr{D}|^{-d+2}=-c \int_{M} \tau \sqrt{g} d x$ (see [26], Theorem 11.2) is so fundamental. Here $\tau$ is the scalar curvature (positive on the sphere) and $c$ is a positive constant.

We give here another proof of this result.

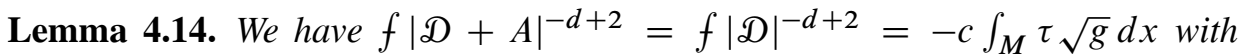

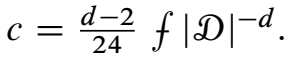

Proof. We get from [19], Lemma 4.10 (ii), the following equality, where $X:=A D+$ $D A+A^{2}$ :

$$
f|\mathscr{D}+A|^{-d+2}-f|\mathscr{D}|^{-d+2}=\frac{(d-2)}{2}\left(\frac{d}{4} f X^{2}|\mathscr{D}|^{-d-2}-f X|\mathscr{D}|^{-d}\right) .
$$

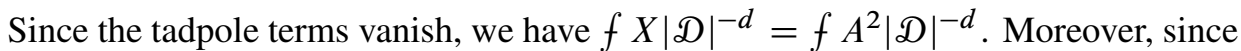
$\bmod \mathrm{OP}^{1}, X^{2}=(A \mathscr{D})^{2}+(\mathscr{D} A)^{2}+A \mathscr{D}^{2} A+\mathscr{D} A^{2} \mathscr{D}$, we get with $\left[\mathscr{D}^{2}, A\right] \in \mathrm{OP}^{1}$,

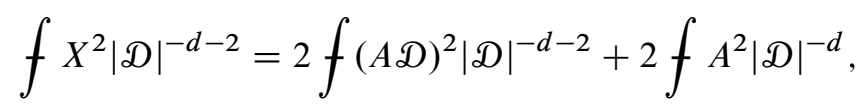

which yields

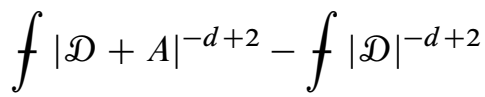

$$
\begin{aligned}
& =\frac{d(d-2)}{4}\left(f(A \mathscr{D})^{2}|\mathscr{D}|^{-d-2}-\frac{2-d}{d} f A^{2}|\mathscr{D}|^{-d}\right) .
\end{aligned}
$$

Thus, it is sufficient to check that

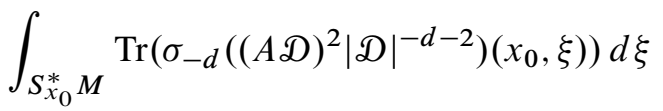

$$
\begin{aligned}
& =\frac{2-d}{d} \int_{S_{x_{0}}^{*} M} \operatorname{Tr}\left(\sigma_{-d}\left(A^{2}|\mathcal{D}|^{-d}\right)\left(x_{0}, \xi\right)\right) d \xi .
\end{aligned}
$$


A straightforward computation yields, with $A=:-i a_{\mu} \gamma^{\mu}$ and $\sigma_{1}^{\mathscr{D}}\left(x_{0}, \xi\right)=$ $\gamma^{\mu} \xi_{\mu}$

$$
\begin{aligned}
& \int_{S_{x_{0}}^{*} M} \sigma_{-d}\left((A D)^{2}|\mathcal{D}|^{-d-2}\right)\left(x_{0}, \xi\right) d \xi=-\frac{1}{d} a_{\mu} a_{\tau} \operatorname{Tr}\left(\gamma^{\mu} \gamma^{\nu} \gamma^{\tau} \gamma_{\nu}\right) \operatorname{Vol}\left(S^{d-1}\right) \text {, } \\
& \int_{S_{x_{0}}^{*} M} \sigma_{-d}\left(A^{2}|\mathcal{D}|^{-d}\right)\left(x_{0}, \xi\right) d \xi=-a_{\mu} a_{\tau} \operatorname{Tr}\left(\gamma^{\mu} \gamma^{\tau}\right) \operatorname{Vol}\left(S^{d-1}\right) .
\end{aligned}
$$

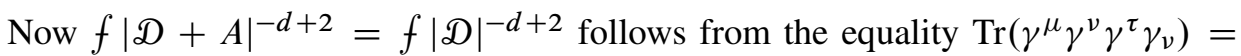
$(2-d) \operatorname{Tr}\left(\gamma^{\mu} \gamma^{\tau}\right)$. The constant $c$ is given in [26], Theorem 11.2 and normalization (11.2).

Remark 4.15. In [17], p. 210, Definition 1.147, the above result justifies the definition of a scalar curvature for $(\mathcal{A}, \mathscr{H}, \mathscr{D})$ as $\mathcal{R}(a):=f a|\mathfrak{D}|^{-d+2}$ for $a \in \mathcal{A}$. This map is of course a trace on $\mathcal{A}$ for a commutative geometry. But for the triple associated to $\mathrm{SU}_{q}(2)$ this not a trace since (see [33])

$$
\mathcal{R}\left(a a^{*}\right)=f a a^{*}|\mathscr{D}|^{-1}=\frac{-q^{4}+6 q^{2}+3}{2\left(1-q^{2}\right)^{2}},
$$

while

$$
\mathcal{R}\left(a^{*} a\right)=f a^{*} a|\mathcal{D}|^{-1}=\frac{3 q^{4}+6 q^{2}-1}{2\left(1-q^{2}\right)^{2}} .
$$

\section{Appendix}

\section{A.1. Pseudodifferential operators}

Definition A.1. Let us define $\mathscr{D}(\mathcal{A})$ as the polynomial algebra generated by $\mathcal{A}$, $J \mathscr{A} J^{-1}, \mathcal{D}$ and $|\mathcal{D}|$.

A pseudodifferential operator is an operator $T$ such that there exists $d \in \mathbb{Z}$ such that, for any $N \in \mathbb{N}$, there exist $p \in \mathbb{N}_{0}, P \in \mathscr{D}(\mathcal{A})$ and $R \in \mathrm{OP}^{-N}(p, P$ and $R$ may depend on $N$ ) with $P D^{-2 p} \in \mathrm{OP}^{d}$ and

$$
T=P D^{-2 p}+R
$$

Define $\Psi(\mathcal{A})$ as the set of pseudodifferential operators and $\Psi(\mathcal{A})^{k}:=\Psi(\mathcal{A}) \cap \mathrm{OP}^{k}$.

Note that the notion of pseudodifferential operator is modified as $\Psi(\mathcal{A})$ now includes $J \mathcal{A}^{-1}$; see [19].

If $A$ is a 1 -form, then $A$ and $J A J^{-1}$ are in $\mathscr{D}(\mathcal{A})$ and moreover $\mathscr{D}(\mathcal{A}) \subseteq$ $\bigcup_{k \in \mathbb{N}_{0}} \mathrm{OP}^{k}$. Since $|\mathscr{D}| \in \mathscr{D}(\mathcal{A})$ by construction and $P_{0}$ is a pseudodifferential 
operator, $|D|^{k}$ is a pseudodifferential operator (in $\mathrm{OP}^{k}$ ) for any $k \in \mathbb{Z}$. Let us also remark that $\mathscr{D}(\mathcal{A}) \subseteq \Psi(\mathcal{A}) \subseteq \bigcup_{k \in \mathbb{Z}} \mathrm{OP}^{k}$.

The set of all pseudodifferential operators $\Psi(\mathcal{A})$ is an algebra. We denote by $\Psi_{1}(\mathcal{A})$ the subalgebra of $\Psi(\mathcal{A})$ defined in the same way as $\Psi(\mathcal{A})$, replacing $\mathscr{D}(A)$ with the polynomial algebra generated by $\mathscr{D}, \mathcal{A}$ and $J \mathcal{A}^{-1}$. This algebra is similar to the one defined in [16].

\section{A.2. Zeta functions and dimension spectrum}

For any operator $B$ and if $X$ is either $D$ or $D_{A}$, we define

$$
\zeta_{X}^{B}(s):=\operatorname{Tr}\left(B|X|^{-s}\right), \quad \zeta_{X}(s):=\operatorname{Tr}\left(|X|^{-s}\right) .
$$

The dimension spectrum $\operatorname{Sd}(\mathcal{A}, \mathcal{H}, \mathcal{D})$ of a spectral triple has been defined in [12], [18]. It is extended here to pay attention to the operator $J$ and to our definition of pseudodifferential operator.

Definition A.1. The spectrum dimension of the spectral triple is to be defined as the subset $\operatorname{Sd}(\mathcal{A}, \mathscr{H}, \mathscr{D})$ of all poles of the functions $\zeta_{\mathscr{D}}^{P}:=s \mapsto \operatorname{Tr}\left(P|\mathscr{D}|^{-s}\right)$ where $P$ is any pseudodifferential operator in $\mathrm{OP}^{0}$. The spectral triple $(\mathcal{A}, \mathscr{H}, \mathscr{D})$ is said to be simple when these poles are all simple.

The following is part of folklore in noncommutative geometry, even if sometimes it is unclear if there is an equality or an inclusion of $\operatorname{Sp}(M)$ in $\{d-k: k \in \mathbb{N}\}$.

Proposition A.2. Let $\mathrm{Sp}(M)$ be the spectrum dimension of a commutative geometry of dimension $d$. Then $\operatorname{Sp}(M)$ is simple and $\operatorname{Sp}(M)=\{d-k: k \in \mathbb{N}\}$.

Proof. Let $a \in \mathcal{A}=C^{\infty}(M)$ such that its trace norm $\|a\|_{\mathscr{L}^{1}}$ is non-zero and let $P_{k}:=a|D|^{-k}$ for $k \in \mathbb{N}$. Then $P_{k} \in \mathrm{OP}^{-k} \subset \mathrm{OP}^{0}$ and its associated zeta-function has a pole at $d-k$ :

$$
\begin{aligned}
\operatorname{Res}_{s=d-k} \zeta_{\mathscr{D}}^{P}(s) & =\operatorname{Res}_{s=0} \zeta_{\mathscr{D}}^{P}(s+d-k) \\
& =\operatorname{Res}_{s=0} \operatorname{Tr}\left(a|\mathscr{D}|^{-k}|\mathscr{D}|^{-(s+d-k)}\right) \\
& =f a|\mathscr{D}|^{-d} \\
& =\int_{M} a(x) \int_{S_{x}^{*} M} \operatorname{Tr}\left(\left(\sigma_{1}^{|D|}\right)^{-d}(x, \xi)\right)|d \xi||d x| \\
& =\int_{M} a(x) \int_{S_{x}^{*} M}\|\xi\|^{-d / 2}|d \xi||d x| \\
& =\int_{M} a(x) v_{g}(x)=\|a\|_{\mathscr{L}^{1}} \neq 0,
\end{aligned}
$$


where $v_{g}$ is the Riemann density normalized on $g$-orthonormal basis of $T M$.

Conversely, since $\Psi(\mathcal{A})^{0}$ is contained in the algebra of all pseudodifferential operators of order less or equal to 0 , it is known (see [29], [51], [52]) that $\operatorname{Sp}(M) \subset$ $\{d-k: k \in \mathbb{N}\}$.

The fact that all poles are simple is due to the fact that $\mathcal{D}$ being differential and $M$ being without boundary, $a_{k}^{\prime}=0$ for all $k \in \mathbb{N}^{*}$ in (15).

\section{A.3. Proof of technical lemmas}

Proof of Lemma 4.7. (i) Let $f \in E_{j}$ and $\alpha \in \mathbb{N}^{d}$. If $f(x, \xi)=\sum_{i \in I} \frac{\xi^{\beta^{i}}}{\|\xi\|_{x}^{2 k_{i}}} h_{i}(x)$ it follows that

$$
\partial_{\xi}^{\alpha} f=\sum_{i \in I} \partial_{\xi}^{\alpha}\left(\frac{\xi^{\beta^{i}}}{\|\xi\|_{x}^{2 k_{i}}}\right) h_{i}(x)=\sum_{i \in I} \sum_{\gamma \leq \alpha}\left(\begin{array}{l}
\alpha \\
\gamma
\end{array}\right) \partial_{\xi}^{\alpha-\gamma}\left(\xi^{\beta^{i}}\right) \partial_{\xi}^{\gamma}\left(\frac{1}{\|\xi\|_{x}^{2 k_{i}}}\right) h_{i}(x) .
$$

We check by induction that we can write

$$
\partial_{\xi}^{\gamma}\left(\frac{1}{\|\xi\|_{x}^{2 k_{i}}}\right)=\frac{1}{\|\xi\|_{x}^{2 k_{i}(|\gamma|+1)}} \sum_{p} \lambda_{p} \prod_{j=1}^{|\gamma|} \partial_{\xi}^{\beta^{j, p}}\|\xi\|_{x}^{2 k_{i}}
$$

where $\lambda_{p}$ are real numbers, the sum on indices $p$ is finite, and $\sum_{j=1}^{|\gamma|} \beta^{j, p}=\gamma$. As a consequence, since $\|\xi\|_{x}^{2 k_{i}}=\left(g^{k l}(x) \xi_{k} \xi_{l}\right)^{k_{i}}$ is a homogeneous polynomial in $\xi$ of degree $2 k_{i}$, we get $\partial_{\xi}^{\alpha} f \in E_{j-|\alpha|}$. The inclusions $E_{j} E_{j^{\prime}} \subseteq E_{j+j^{\prime}}, \partial_{x}^{\alpha} E_{j} \subseteq E_{j}$ are straightforward.

(ii) The proof is similar to $(i)$ since by induction

$$
\partial_{\xi}^{\gamma}\left(\frac{1}{\|\xi\|_{x}}\right)=\frac{1}{\|\xi\|_{x}^{2|\gamma|+1}} \sum_{p} \lambda_{p} \prod_{j=1}^{|\gamma|} \partial_{\xi}^{\beta^{j, p}}\|\xi\|_{x}^{2}
$$

where $\lambda_{p}$ are real numbers, the sum over the indices $p$ is finite and $\sum_{j=1}^{|\gamma|} \beta^{j, p}=\gamma$.

(iii) Straightforward.

(iv) The product symbol formula for two classical pseudodifferential operators $P \in \Psi^{p}(M), Q \in \Psi^{q}(M)$ gives

$$
\sigma_{p+q-j}^{P Q}=\sum_{\alpha \in \mathbb{N}^{d}} \sum_{k \geq 0,|\alpha|+k \leq j} i^{|\alpha|} \frac{(-1)^{|\alpha|}}{\alpha !} \partial_{\xi}^{\alpha} \sigma_{p-j+|\alpha|+k}^{P} \partial_{x}^{\alpha} \sigma_{q-k}^{Q}
$$

It is the presence of the factor $i^{|\alpha|}$ that will be crucial in later arguments like, e.g., in Lemma 4.9. 
If $P, Q \in \mathscr{B}_{\mathrm{e}}$, then (i) implies that $\partial_{\xi}^{\alpha} \sigma_{p-j+|\alpha|+k}^{P} \in E_{p-j+k}$ and $\partial_{x}^{\alpha} \sigma_{q-k}^{Q} \in E_{q-k}$. Again by (i), we obtain $\partial_{\xi}^{\alpha} \sigma_{p-j+|\alpha|+k}^{P} \partial_{x}^{\alpha} \sigma_{q-k}^{Q} \in E_{p+q-j}$, so the result follows from (A.1).

(v) A similar argument as (iv) can be applied, using (ii) to obtain $\mathcal{B}_{\mathrm{o}} \mathscr{B}_{\mathrm{o}} \subseteq \mathcal{B}_{\mathrm{e}}$ and (iii) to get $\mathscr{B}_{0} \mathscr{B}_{\mathrm{e}} \subseteq \mathscr{B}_{\mathrm{o}}, \mathscr{B}_{\mathrm{e}} \mathscr{B}_{\mathrm{o}} \subseteq \mathscr{B}_{\mathrm{o}}$.

Proof of Lemma 4.8. Assume $P \in \mathscr{B}_{\mathrm{e}}$ so $p$ is even. From the parametrix equation $P P^{-1}=1$, we obtain $\sigma_{-p}^{P^{-1}}=\left(\sigma_{p}^{P}\right)^{-1}=\|\xi\|_{x}^{-p} \in E_{-p}$. Moreover, using (A.1), we see that

$$
\begin{aligned}
\sigma_{-p-j}^{P^{-1}}=- & \left(\sigma_{p}^{P}\right)^{-1}\left(\sum_{0 \leq k<j} \sigma_{p-j+k}^{P} \sigma_{-p-k}^{P^{-1}}\right. \\
& \left.+\sum_{0<|\alpha| \leq j} \sum_{k=0}^{j-|\alpha|} i^{|\alpha|} \frac{(-1)^{|\alpha|}}{\alpha !} \partial_{\xi}^{\alpha} \sigma_{p-j+|\alpha|+k}^{P} \partial_{x}^{\alpha} \sigma_{-p-k}^{P^{-1}}\right)
\end{aligned}
$$

for any $j \in \mathbb{N}^{*}$. We prove by induction that $\sigma_{-p-j}^{P^{-1}} \in E_{-p-j}$ for any $j \in \mathbb{N}$ : suppose that for $j \in \mathbb{N}^{*}$ we have $\sigma_{-p-j^{\prime}}^{P^{-1}} \in E_{-p-j^{\prime}}$ for any $j^{\prime}<j$. We then directly check with Lemma 4.7 and (A.2) that $\sigma_{-p-j}^{P^{-1}} \in E_{-p-j}$.

The case $P \in \mathscr{B}_{0}$ is similar.

(ii) Since $\mathscr{D} \in \mathscr{B}_{\mathrm{e}}, \mathscr{D}^{-2}$ is in $\mathscr{B}_{\mathrm{e}}$ by $(i)$ and 4.7 and so is $\mathscr{D}^{k}$.

Using (A.1) for the equation $|\mathscr{D}||\mathscr{D}|=\mathscr{D}^{2}$, we check that $\sigma_{1}^{|\mathscr{D}|}(x, \xi)=\|\xi\|_{x}$ and

$$
\begin{aligned}
\sigma_{1-j}^{|\mathcal{D}|}= & \frac{1}{2\|\xi\|_{x}}\left(\sigma_{2-j}^{\mathcal{D}^{2}}-\sum_{0<k<j} \sigma_{1-j+k}^{|\mathcal{D}|} \sigma_{1-k}^{|\mathcal{D}|}\right. \\
& \left.+\sum_{0<|\alpha| \leq j} \sum_{k=0}^{j-|\alpha|} i^{|\alpha|} \frac{(-1)^{|\alpha|}}{\alpha !} \partial_{\xi}^{\alpha} \sigma_{1-j+|\alpha|+k}^{|\mathcal{D}|} \partial_{x}^{\alpha} \sigma_{1-k}^{|\mathcal{D}|}\right)
\end{aligned}
$$

for any $j \in \mathbb{N}^{*}$. Again, a straightforward induction argument shows that $\sigma_{1-j}^{|\mathcal{D}|} \in$ $O_{1-j}$ for any $j \in \mathbb{N}$, and thus $|\mathscr{D}| \in \mathscr{B}_{0}$. The result now follows as above.

(iii) Since $\sigma_{-d}^{P} \in E_{-d}$, we have $\sigma_{-d}^{P}(x, \xi)=\sum_{i \in I} \frac{\xi^{\beta^{i}}}{\|\xi\|_{x}^{2 k_{i}}} h_{i}(x)$, where the $\left|\beta^{i}\right|$ are odd. The integration on the cosphere in (14) therefore vanishes.

(iv) The same argument can be applied.

(v) Direct consequence of (iii) and (iv).

Proof of Lemma 4.9. (i) Consequence of (A.1).

(ii) Consequence of (A.2).

(iii) It is clear that $\mathscr{D} \in \mathcal{C}$ and the fact that $|\mathscr{D}| \in \mathcal{C}$ follows from (A.3).

Acknowledgments. We thank Jean-Marie Lescure, Thomas Schücker and Dmitri Vassilevich for helpful discussions. 


\section{References}

[1] T. Ackermann, A note on the Wodzicki residue. J. Geom. Phys. 20 (1996), 404-406. Zbl 0864.58057 MR 1419429

[2] M.Adler, On a trace functional for formal pseudo differential operators and the symplectic structure of the Korteweg-de Vries type equations. Invent. Math. 50 (1978/79), 219-248. Zbl 0393.35058 MR 520927

[3] T. P. Branson and P. B. Gilkey, The asymptotics of the Laplacian on a manifold with boundary. Comm. Partial Differential Equations 15 (1990), 245-272. Zbl 0721.58052 MR 1032631

[4] T. P. Branson and P. B. Gilkey, Residues of the eta function for an operator of Dirac type with local boundary conditions. Differential Geom. Appl. 2 (1992), 249-267. Zbl 0742.58054 MR 1245326

[5] T. P. Branson, P. B. Gilkey, K. Kirsten, and D. V. Vassilevich, Heat kernel asymptotics with mixed boundary conditions. Nuclear Phys. B 563 (1999), 603-626. Zbl 0955.58021 MR 1731341

[6] L. Carminati, B. Iochum, and T. Schücker, Noncommutative Yang-Mills and noncommutative relativity: a bridge over troubled water. Eur. Phys. J. C Part. Fields 8 (1999), 697-709. MR 1738959

[7] A. H. Chamseddine and A. Connes, The spectral action principle. Comm. Math. Phys. 186 (1997), 731-750. Zbl 0894.58007 MR 1463819

[8] A. H. Chamseddine and A. Connes, Quantum gravity boundary terms from the spectral action of noncommutative space. Phys. Rev. Lett. 99 (2007), 071302. MR 2338507

[9] A. H. Chamseddine, A. Connes, and M. Marcolli, Gravity and the standard model with neutrino mixing. Adv. Theor. Math. Phys. 11 (2007), 991-1089. Zbl 1140.81022 MR 2368941

[10] A. Connes, The action functional in noncommutative geometry. Comm. Math. Phys. 117 (1988), 673-683. Zbl 0658.53068 MR 953826

[11] A. Connes, Noncommutative geometry. Academic Press, San Diego, CA, 1994. Zbl 0818.46076 MR 1303779

[12] A. Connes, Geometry from the spectral point of view. Lett. Math. Phys. 34 (1995), 203-238. Zbl 1042.46515 MR 1345552

[13] A. Connes, Noncommutative geometry and reality. J. Math. Phys. 36 (1995), 6194-6231. Zbl 0871.58008 MR 1355905

[14] A. Connes, Gravity coupled with matter and the foundation of non-commutative geometry. Comm. Math. Phys. 182 (1996), 155-176. Zbl 0881.58009 MR 1441908

[15] A. Connes, On the spectral characterization of manifolds. Preprint 2008. arXiv:0810.2088

[16] A. Connes and A. H. Chamseddine, Inner fluctuations of the spectral action. J. Geom. Phys. 57 (2006), 1-21. Zbl 1105.58004 MR 2265456

[17] A. Connes and M. Marcolli, Noncommutative geometry, quantum fields and motives. Amer. Math. Soc. Colloq. Publ. 55, Amer. Math. Soc., Providence, RI, 2008. Zbl 1209.58007 MR 2371808 
[18] A. Connes and H. Moscovici, The local index formula in noncommutative geometry. Geom. Funct. Anal. 5 (1995), 174-243. Zbl 0960.46048 MR 1334867

[19] D. Essouabri, B. Iochum, C. Levy, and A. Sitarz, Spectral action on noncommutative torus. J. Noncommut. Geom. 2 (2008), 53-123. Zbl 1156.46046 MR 2366124

[20] B. V. Fedosov, F. Golse, E. Leichtnam, and E. Schrohe, The noncommutative residue for manifolds with boundary. J. Funct. Anal. 142 (1996), 1-31.Zbl 0877.58005 MR 1419415

[21] V. Gayral and B. Iochum, The spectral action for Moyal phase. J. Math. Phys. 46 (2005), 043503. Zbl 1067.58020 MR 2131255

[22] V. Gayral, B. Iochum, and J. C. Várilly, Dixmier traces on noncompact isospectral deformations. J. Funct. Anal. 237 (2006), 507-539. Zbl 1106.46054 MR 2230348

[23] V. Gayral, B. Iochum, and D. V. Vassilevich, Heat kernel and number theory on NC-torus. Comm. Math. Phys. 273 (2007), 415-443. Zbl 1124.58010 MR 2318313

[24] P. B. Gilkey, Invariance theory, the heat equation, and the Atiyah-Singer index theorem. 2nd ed., Stud. Adv. Math., CRC Press, Boca Raton, FL, 1995. Zbl 0856.58001 MR 1396308

[25] P. B. Gilkey, Asymptotic formulae in spectral geometry. Stud. Adv. Math., Chapman \& Hall/CRC, Boca Raton, FL, 2004. Zbl 1080.58023 MR 2040963

[26] J. M. Gracia-Bondía, J. C. Várilly, and H. Figueroa, Elements of noncommutative geometry. Birkhäuser Adv. Texts, Birkhäuser, Boston 2001. Zbl 0958.46039 MR 1789831

[27] G. Grubb and E. Schrohe, Trace expansions and the noncommutative residue for manifolds with boundary. J. Reine Angew. Math. 536 (2001), 167-207. Zbl 0980.58017 MR 1837429

[28] G. Grubb and R. T. Seeley, Weakly parametric pseudodifferential operators and AtiyahPatodi-Singer boundary problems. Invent. Math. 121 (1995), 481-529. Zbl 0851.58043 MR 1353307

[29] V. Guillemin, A new proof of Weyl's formula on the asymptotic distribution of eigenvalues. Adv. in Math. 55 (1985), 131-160. Zbl 0559.58025 MR 772612

[30] S. W. Hawking, Zeta function regularization of path integrals in curved spacetime. Comm. Math. Phys. 55 (1977), 133-148. Zbl 0407.58024 MR 0524257

[31] N. Higson, The local index formula in noncommutative geometry. ICTP Lecture Notes 15, Abdus Salam Internat. Cent. Theoret. Phys., Trieste 2004. Zbl 1122.58015 MR 2175637

[32] B. Iochum and C. Levy, Spectral triples and manifolds with boundaries. Preprint 2010. arXiv: 1001.3927

[33] B. Iochum, C. Levy, and A. Sitarz, Spectral action on $\mathrm{SU}_{q}(2)$. Comm. Math. Phys. 289 (2009), 107-155. Zbl 1173.46053 MR 2504847

[34] W. Kalau and M. Walze, Gravity, non-commutative geometry and the Wodzicki residue. J. Geom. Phys. 16 (1995), 327-344. Zbl 0826.58008 MR 1336738

[35] C. Kassel, Le résidu non commutatif (d'après M. Wodzicki). Sém. Bourbaki, Vol. 1988/89, Exp. No. 708, Astérisque 177-178 (1989), 199-229. Zbl 0701.58054 MR 1040574

[36] D. Kastler, The Dirac operator and gravitation. Comm. Math. Phys. 166 (1995), 633-643. Zbl 0823.58046 MR 1312438 
[37] M. Knecht and T. Schücker, Spectral action and big desert. Phys. Lett. B 640 (2006), 272-277. MR 2275783

[38] T. Krajewski, Classification of finite spectral triples. J. Geom. Phys. 28 (1998), 1-30. Zbl 0932.58006 MR 1653118

[39] M. Lesch, On the noncommutative residue for pseudodifferential operators with log-polyhomogeneous symbols. Ann. Global Anal. Geom. 17 (1999), 151-187. Zbl 0920.58047 MR 1675408

[40] J.-M. Lescure, Triplets spectraux pour les variétés à singularité conique isolée. Bull. Soc. Math. France 129 (2001), 593-623. Zbl 1029.19002 MR 1894151

[41] J.-M. Lescure and S. Paycha, Uniqueness of multiplicative determinants on elliptic pseudodifferential operators. Proc. Lond. Math. Soc. (3) 94 (2007), 772-812. Zbl 1193.58018 MR 2325320

[42] Yu. I. Manin, Algebraic aspects of nonlinear differential equations. J. Soviet Math. Sci. 11 (1979), 1-122. Zbl 0419.35001 MR 0501136

[43] R. Ponge, A new short proof of the local index formula and some of its applications. Comm. Math. Phys. 241 (2003), 215-234; Erratum, ibid. 248(2004), 639. Zbl 1191.58006 MR 2013798

[44] R. Ponge, Noncommutative geometry and lower dimensional volumes in Riemannian geometry. Lett. Math. Phys. 83 (2008), 19-32. Zbl 1136.58017 MR 2377943

[45] E. Schrohe, Noncommutative residues and manifolds with conical singularities. J. Funct. Anal. 150 (1997), 146-174. Zbl 0903.58057 MR 1473629

[46] E. Schrohe, Noncommutative residues, Dixmier's trace, and heat trace expansions on manifolds with boundary. In Geometric aspects of partial differential equations. Contemp. Math. 242, Amer. Math. Soc. Providence, R.I., 1999, 161-186. Zbl 0960.58013 MR 1714485

[47] F. J. Vanhecke, On the product of real spectral triples. Lett. Math. Phys. 50 (1999), 157162. Zbl 1079.58507 MR 1761173

[48] D. V. Vassilevich, Heat kernel expansion: user's manual. Phys. Rep. 388 (2003), 279-360. Zbl 1042.81093 MR 2017528

[49] Y. Wang, Gravity and the noncommutative residue for manifolds with boundary. Lett. Math. Phys. 80 (2007), 37-56. Zbl 1127.58025 MR 2314843

[50] M. Wodzicki, Spectral asymmetry and local invariants (in Russian). Habilitation thesis, Steklov Math. Inst., Moscow 1984.

[51] M. Wodzicki, Local invariants of spectral asymmetry. Invent. Math. 75 (1984), 143-177. Zbl 0538.58038 MR 728144

[52] M. Wodzicki, Noncommutative residue. I. Fundamentals. In $K$-theory, arithmetic and geometry (Moscow, 1984-1986), Lecture Notes in Math. 1289, Springer, Berlin 1987, 320-399. Zbl 0649.58033 MR 0923140

Received April 10, 2009; revised October 13, 2009 
B. Iochum, UMR 6207, Unité Mixte de Recherche du CNRS et des Universités AixMarseille I, Aix-Marseille II et de l'Université du Sud Toulon-Var, Laboratoire affilié à la FRUMAM - FR 2291 and Université de Provence, France

E-mail: iochum@cpt.univ-mrs.fr

C. Levy, UMR 6207, Unité Mixte de Recherche du CNRS et des Universités Aix-Marseille I, Aix-Marseille II et de l'Université du Sud Toulon-Var, Laboratoire affilié à la FRUMAM - FR 2291 and Université de Provence, France

E-mail: levy@cpt.univ-mrs.fr 